\title{
A method of convergence acceleration of some continued fractions II
}

\author{
Rafal Nowak
}

Received: 11 March 2012 / Accepted: 27 August 2012 / Published online: 19 September 2012 (C) The Author(s) 2012. This article is published with open access at SpringerLink.com

\begin{abstract}
Most of the methods for convergence acceleration of continued fractions $\mathbf{K}\left(a_{m} / b_{m}\right)$ are based on the use of modified approximants $S_{m}\left(\omega_{m}\right)$ in place of the classical ones $S_{m}(0)$, where $\omega_{m}$ are close to the tails $f^{(m)}$ of the continued fraction. Recently (Nowak, Numer Algorithms 41(3):297-317, 2006), the author proposed an iterative method producing tail approximations whose asymptotic expansion accuracies are being improved in each step. This method can be successfully applied to a convergent continued fraction $\mathbf{K}\left(a_{m} / b_{m}\right)$, where $a_{m}=\alpha_{-2} m^{2}+\alpha_{-1} m+\ldots, b_{m}=\beta_{-1} m+\beta_{0}+\ldots\left(\alpha_{-2} \neq 0\right.$, $\left|\beta_{-1}\right|^{2}+\left|\beta_{0}\right|^{2} \neq 0$, i.e. $\left.\operatorname{deg} a_{m}=2, \operatorname{deg} b_{m} \in\{0,1\}\right)$. The purpose of this paper is to extend this idea to the class of two-variant continued fractions $\mathbf{K}\left(a_{n} / b_{n}+\right.$ $\left.a_{n}^{\prime} / b_{n}^{\prime}\right)$ with $a_{n}, a_{n}^{\prime}, b_{n}, b_{n}^{\prime}$ being rational in $n$ and $\operatorname{deg} a_{n}=\operatorname{deg} a_{n}^{\prime}, \operatorname{deg} b_{n}=$ $\operatorname{deg} b_{n}^{\prime}$. We give examples involving continued fraction expansions of some elementary and special mathematical functions.
\end{abstract}

Keywords Convergence acceleration • Continued fraction • Tail • Modified approximant

Mathematics Subject Classifications (2010) $65 \mathrm{~B} 99 \cdot 33 \mathrm{~F} 05$

\footnotetext{
R. Nowak $(\bowtie)$

Institute of Computer Science, University of Wrocław, ul. Joliot-Curie 15, 50-383 Wrocław, Poland

e-mail: rno@cs.uni.wroc.pl
} 


\section{Introduction}

Many valued mathematical constants and elementary or special functions have continued fraction expansions. For example, the following continued fraction (CF, in short)

$$
\frac{\arcsin x}{\sqrt{1-x^{2}}}=\frac{x}{\mid 1}+\underset{n=1}{\mathbf{K}}\left[\frac{-\left(4 n^{2}-2 n\right) x^{2} \mid}{4 n-1}+\frac{-\left(4 n^{2}-2 n\right) x^{2}}{\mid 4 n+1}\right], \quad \pm x \notin[1,+\infty),
$$

is very useful expansion of the well-known inverse trigonometric function; see, e.g., [13, p. 155, Eq. 18] or [3, p. 205, Eq. 11.4.5]. To compute the value of (1) or, in general, the value of $\mathrm{CF}$ of the form

$$
b_{0}^{\prime}+\underset{n=1}{\mathbf{K}}\left[\frac{a_{n} \mid}{\mid b_{n}}+\frac{a_{n}^{\prime} \mid}{\mid b_{n}^{\prime}}\right],
$$

one can use the sequence $\left\{S_{n}(0)\right\}$ of its classical approximants; see $[8$, pp. 7, 56] or [9, p. 5, Eq. 1.2.1] for more details. Putting $x=\sqrt{2} / 2$ in (1) yields a quite fast convergent method of computation of $\pi$ constant. Namely, using the approximant $S_{20}(0)$ one can obtain almost 16 exact significant decimal digits of the limit. However, in some cases, the convergence of the sequence of classical approximants of $\mathrm{CF}$ (2) (the convergence of $\mathrm{CF}$, in short) can be extremely slow. It is so for CF (1) if $x \approx \pm 1$. In such a case, application of modified approximants $S_{n}(\omega)$ :

$$
\begin{aligned}
& S_{0}(\omega)=b_{0}^{\prime}+\omega, \\
& S_{2 n-1}(\omega)=b_{0}^{\prime}+\stackrel{\mathbf{K}}{k=1}_{\mathbf{K}-1}\left[\begin{array}{c}
a_{k} \mid \\
{\left[b_{k}\right.}
\end{array}+\frac{a_{k}^{\prime} \mid}{\mid b_{k}^{\prime}}\right]+\frac{a_{n} \mid}{\mid b_{n}+\omega}, \quad n \in \mathbb{N}, \\
& S_{2 n}(\omega)=b_{0}^{\prime}+\stackrel{\mathbf{K}}{k=1}_{n-1}\left[\frac{a_{k} \mid}{\mid b_{k}}+\frac{a_{k}^{\prime} \mid}{\mid b_{k}^{\prime}}\right]+\frac{\left.a_{n}\right\rfloor}{\mid b_{n}}+\frac{a_{n}^{\prime}}{\mid b_{n}^{\prime}+\omega}, \quad n \in \mathbb{N},
\end{aligned}
$$

may be much helpful in computing the value of CF.

We use the symbol $f^{(m)}$ to denote the $m$-th tail of CF (2):

$$
\begin{array}{rlrl}
f^{(2 n-1)} & =\frac{\left.a_{n}^{\prime}\right\rfloor}{\mid b_{n}^{\prime}}+\underset{k=n+1}{\mathbf{K}}\left[\frac{a_{k} \mid}{\mid b_{k}}+\frac{a_{k}^{\prime} \mid}{\mid b_{k}^{\prime}}\right], & & n \in \mathbb{N}, \\
f^{(2 n)} & =\underset{k=n+1}{\mathbf{K}}\left[\begin{array}{c}
a_{k} \mid \\
\mid b_{k}
\end{array}+\frac{a_{k}^{\prime} \mid}{\mid b_{k}^{\prime}}\right], & n \in \mathbb{N} \cup\{0\} .
\end{array}
$$

Most of the methods for convergence acceleration of CFs are based on the use of modified approximants $S_{m}\left(\omega_{m}\right)$, where modifying factor $\omega_{m}$ is close to the tail $f^{(m)}$; see, e.g. [4, 5, 7, 8, 10-12]. For example, the good approximations of the tails of CF in (1) can be obtained using so-called 'improvement machine'the method of Lorentzen and Waadeland, since its can be transformed equivalently into a limit periodic CF of loxodromic type; see [7] or [9, p. 227] for more details. One can also verify that the even part of CF in (1) can be effectively computed using 'plus method'-one of the methods given in [10], where the convergence acceleration of $\mathrm{CFs} \in \mathcal{C}_{20} \cup \mathcal{C}_{21}$ was proposed; $\mathcal{C}_{k l}$ means the class 
of one-variant CFs of the form $\mathbf{K}\left(a_{m} / b_{m}\right)$ with $a_{m}$ and $b_{m}$ being rational functions in $m$ :

$$
a_{m}=\alpha_{-k} m^{k}+\alpha_{1-k} m^{k-1}+\ldots, \quad b_{m}=\beta_{-l} m^{l}+\beta_{1-l} m^{l-1}+\ldots .
$$

In the case of CFs of the form (2), the performance of the methods in [10] can be increased, if one not contract the $\mathrm{CF}$ into even or odd part, but take into account the two-variant character of $\mathrm{CF}$.

In this paper, we extend the results in [10] to the class of so-called twovariant $\mathrm{CFs} \in \mathcal{D}_{k l}$, i.e. of the form (2), where

$$
\begin{array}{ll}
a_{n}=p_{-k} n^{k}+p_{1-k} n^{k-1}+\ldots, & a_{n}^{\prime}=p_{-k}^{\prime} n^{k}+p_{1-k}^{\prime} n^{k-1}+\ldots, \\
b_{n}=q_{-l} n^{k}+q_{1-l} n^{l-1}+\ldots, & b_{n}^{\prime}=q_{-l}^{\prime} n^{k}+q_{1-l}^{\prime} n^{l-1}+\ldots
\end{array}
$$

and $p_{k}, p_{k}^{\prime}, q_{l}, q_{l}^{\prime} \neq 0(k, l \in \mathbb{Z})$. The initial coefficients $p_{i}, p_{i}^{\prime}, q_{j}, q_{j}^{\prime}$ and the numbers $k, l$ should satisfy some additional conditions, sufficient for the convergence of CF; see Section 2. We allow the numerators $a_{n}, a_{n}^{\prime}$ and the denominators $b_{n}, b_{n}^{\prime}$ to be complex. One can find a lot of examples involving CFs $\in \mathcal{D}_{k l}$ in Perron's book [13]. More CF expansions can be found in (chronological order): [9, 16] and [3]. The authors of [3] have developed the website which shows the applications of continued fractions to approximation of many special functions (see http://www.cfhblive.ua.ac.be/).

For example, the following CF expansion holds:

$$
\int_{0}^{\infty} e^{-t x} \operatorname{cn}(t ; k) d t=\frac{1}{\mid x}+\underset{n=1}{\mathbf{K}}\left[\frac{(2 n-1)^{2} \mid}{\left.\mid \frac{(2 n)^{2} k^{2}}{x}\right], \quad 0<k<1, x>0,}\right.
$$

where $\operatorname{cn}(t ; k)$ is the Jacobi elliptic function, very often applied in electroand magnetostatics, fluid dynamics, as well as nonlinear integral equations (see [13, p. 220, Eq. 15] or [9, p. 283]). Although the (5) is known for almost 100 years (the first edition of Perron's book [13] was issued in 1913), current computer algebra systems do not use it. For instance, the Maple ${ }^{\mathrm{TM}}$ system is not able to compute the integral in the (5) with $x=0.8$ and $k=0.9$; if we replace the upper limit $\infty$ by 500, then the correct result (provided Maple ${ }^{\mathrm{TM}}$ the 32-digit arithmetic precision) is obtained after about $2 \mathrm{~min}$ of waiting on AMD Quad Core Phenom ${ }^{\mathrm{TM}} 1.25 \mathrm{GHz}$ computer. We used Maple ${ }^{\mathrm{TM}} 16$ the latest version of Maple ${ }^{\mathrm{TM}}$ software. Let us note that Maple ${ }^{\mathrm{TM}} 14$ was not able to compute the integral of the form (5) with upper limit replaced by any finite number; the computation was interrupted after $1 \mathrm{~GB}$ of memory was used. In addition, Matlab ${ }^{\circledR}$ (ver. 7.12, R2011a) cannot even compute the good approximation of the Jacobi function $\mathrm{cn}(t, k)$. This shows that the problems which require computing a lot of integrals as in (5) are almost insolvable using the current mathematical software. The motivation for using aforementioned techniques of convergence acceleration of CFs is, among others, the fact that CF expansion (5) allows us to compute such integrals in a split second.

Our main result is a new method for convergence acceleration of CFs $\in \mathcal{D}_{k l}$. In this paper, we consider only $k \in\{1,2\}$ and $l \in\{0,1\}$, since any CF of the class $\mathcal{D}_{k+2, l+1}$ is equivalent to some of the class $\mathcal{D}_{k l}$; see, e.g., [8, p. 73, Theorem. 9] 
for more details on equivalent transformation of $\mathrm{CF}$. We analyse the odd tails $f^{(2 n-1)}$ and derive their asymptotic expansion of the form

$$
f^{(2 n-1)}=\tau_{-\nu} n^{\nu / 2}+\tau_{1-\nu} n^{\nu / 2-1 / 2}+\tau_{2-\nu} n^{\nu / 2-1}+\tau_{3-\nu} n^{\nu / 2-3 / 2}+\ldots
$$

In the case of one-variant $\mathrm{CFs} \in \mathcal{C}_{k l}$, this is close to the method of Hautot [4]; he used several values of initial coefficients $\tau_{j}$ in order to get modifying factors $\omega_{m}$. Then, a standard sequence transformation, such as Brezinki's $\theta$ or $u$-Levin algorithm, was applied to $\left\{S_{m}\left(\omega_{m}\right)\right\}$. Paszkowski [11] showed that such classic sequence transformations may be unsuitable to the character of approximants of $\mathrm{CF}$. He proposed the iterative method producing modifying factors which accuracy increases in each step. The idea of the method in [10] is quite similar, but there were not used calculations of square roots. In this paper we continue this idea and deal with two-variant CFs (2).

In Section 2, we describe the class $\mathcal{D}$ of considered CFs, while in Section 3 we analyse the asymptotic behaviour of their tails. We give some initial coefficients of the asymptotic expansion (6) explicitly (see Corollary 3.3), in order to find starting modifying factors for our method. In Section 4, we describe the method in detail. The main result is in Theorem 4.1 after which we summarize our method in Algorithm 4.2. Finally, in Section 5, we report the results of experiments involving some $\mathrm{CFs} \in \mathcal{D}$.

\section{Class $\mathcal{D}$}

We consider some subclasses of CFs $\in \mathcal{D}_{k l}(k \in\{1,2\}, l \in\{0,1\})$ of the form (2) satisfying

$$
\begin{array}{rlrl}
a_{n} & =p_{-2} n^{2}+p_{-1} n+p_{0} \ldots, & & a_{n}^{\prime}=p_{-2}^{\prime} n^{2}+p_{-1}^{\prime} n+p_{0}^{\prime} \ldots, \\
b_{n}=q_{-1} n+q_{0}+\ldots, & b_{n}^{\prime}=q_{-1}^{\prime} n+q_{0}^{\prime}+\ldots
\end{array}
$$

Let us use the notation (see Table 1) in order to characterize all of them. In this paper, we consider the CFs belonging to the class

$$
\mathcal{D}:=\mathcal{D}_{10}^{=} \cup \mathcal{D}_{10}^{\neq} \cup \mathcal{D}_{11} \cup \mathcal{D}_{20}^{=} \cup \mathcal{D}_{20}^{\neq} \cup \widetilde{\mathcal{D}}_{21}
$$

Table 1 Considered subclasses of CFs

\begin{tabular}{ll}
\hline Subclass symbol & Characterization \\
\hline $\mathcal{D}_{10}^{=} \subset \mathcal{D}_{10}$ & $p_{-1}=p_{-1}^{\prime}$ and $\frac{q_{0} q_{0}^{\prime}}{p_{-1}} \notin(-\infty, 0]$ \\
$\mathcal{D}_{10}^{\neq} \subset \mathcal{C}_{10}$ & $\left|p_{-1}^{\prime}\right| \neq\left|p_{-1}\right|$ \\
$\mathcal{D}_{20}^{=} \subset \mathcal{D}_{20}$ & $p_{-2}=p_{-2}^{\prime}$ and $\frac{\beta^{2}-4 \alpha \gamma}{p_{-2}^{2}} \notin(-\infty, 0]$, \\
$\mathcal{D}_{20}^{\neq} \subset \mathcal{C}_{20}$ & where $\alpha=q_{0}^{\prime}, \beta=p_{-2}+p_{-1}-p_{-1}^{\prime}, \gamma=-p_{-2} q_{0}$ \\
$\widetilde{\mathcal{D}}_{21} \subset \mathcal{D}_{21}$ & $\left|p_{-2}^{\prime}\right| \neq\left|p_{-2}\right|$ \\
& The roots $x_{0}, x_{1}$ of equation $x=\frac{p_{-2}^{\prime}}{q_{-1}^{\prime}+\frac{p}{q_{-1}+x}}$ \\
\hline
\end{tabular}


One can check that $\mathrm{CF}$ in (1) belongs to $\widetilde{\mathcal{D}}_{21}$, while the one in (5)-to the subclass $\mathcal{D}_{20}^{\neq}$.

The class $\mathcal{D}$ is quite wide since it covers almost all of the examples given in the book [3] which comprises with lot of CF expansions of very known mathematical constants, as well as elementary and special functions; see Part III of the book. It does not cover only the subclass of so-called $q$-CFs, where the numerators and denominators $a_{n}, a_{n}^{\prime}, b_{n}, b_{n}^{\prime}$ (cf. (2)) are expressed in terms of $q^{n}$ (see, e.g., [3, Section 19]). One can also find some applications of $k$-variant CFs $\notin \mathcal{D}$ with $k>2$ (see, e.g., [3, p. 236, Eq. 12.5.3]). In such a case one may try to consider the even or odd contraction.

\section{Asymptotic behaviour of the tails}

In this section, we study the asymptotic properties of the tails $f^{(m)}$ of CFs $\in \mathcal{D}$. From definition (4) they satisfy the following two recurrence relations:

$$
f^{(2 n-1)}=\frac{a_{n}^{\prime}}{b_{n}^{\prime}+f^{(2 n)}}, \quad f^{(2 n)}=\frac{a_{n+1}}{b_{n+1}+f^{(2 n+1)}} .
$$

Taking into account the 'two-variant' character of considered CFs, we deal with their odd tails only, i.e.

$$
u_{n}:=f^{(2 n-1)}, \quad n \in \mathbb{N},
$$

and hence we have that

$$
u_{n}=\frac{a_{n}^{\prime}}{b_{n}^{\prime}+\frac{a_{n+1}}{b_{n+1}+u_{n+1}}} .
$$

Therefore, the sequence $\left\{u_{n}\right\}$ is a solution of the following bilinear equation (see also [12, Eq. 1.6]):

$$
b_{n}^{\prime} X_{n} X_{n+1}+\left(a_{n+1}+b_{n+1} b_{n}^{\prime}\right) X_{n}-a_{n}^{\prime} X_{n+1}-a_{n}^{\prime} b_{n+1}=0 .
$$

Since all the coefficients in (10) have asymptotic expansions in powers of $n^{-1}$ (cf. (7)), its solutions have asymptotic expansion in powers of $n^{-1 / 2}$. Moreover, in the case of $\mathrm{CF} \in \mathcal{D}$, using (7) and comparing the term $X_{n} X_{n+1}$ in (10) with others, we conclude that this expansion is at most $\mathcal{O}\left(n^{2}\right)$, i.e.

$$
X_{n}=\tau_{-4} n^{2}+\tau_{-3} n^{3 / 2}+\tau_{-2} n+\tau_{-1} n^{1 / 2}+\tau_{0}+\tau_{1} n^{-1 / 2}+\ldots
$$

However, the bilinear equation (10) has two solutions of the form (11). According to Paszkowski [12, p. 196], for the solution $X_{n}$ we compute $Y_{n}=$ $a_{n+1} /\left(b_{n+1}+X_{n+1}\right)$ and analyse the product $I_{n}:=X_{n} Y_{n}$. The sequence $\left\{u_{n}\right\}$ corresponds to such solution, that this product has asymptotically smaller modulus. This result is related to Pincherle's theorem (see [14] and its generalization [6, Theorem 5.7, p. 164]); see also the works of: Birkhoff [1] and Birkhoff and Trjitzinsky [2]. 
In the sequel, we investigate the asymptotic behaviour of the product $I_{n}$ considering both the solutions of the recurrence (10). The following analysis derives a suitable choice of the coefficients $\tau_{j}$, such that

$$
u_{n}=\tau_{-v} n^{\nu / 2}+\tau_{1-\nu} n^{\nu / 2-1 / 2}+\tau_{2-v} n^{\nu / 2-1}+\tau_{3-v} n^{\nu / 2-3 / 2}+\ldots
$$

We put the expansions (7) and (12) into the recurrence relation (10). After some rearrangement of terms, we obtain an infinite triangular system of equations, satisfied by the coefficients $\tau_{j}$. One can verify that the first equation is the following quadratic equation, satisfied by the coefficient $\tau_{-\nu}$ :

$$
\alpha \tau^{2}+\beta \tau+\gamma=0,
$$

where $\alpha, \beta, \gamma$ are given in Table 2. Once we fix its solution, we simply derive the next coefficients by solving the consecutive equations of the system. This process is rather technical and requires to study all the considered CFs subclasses, separately. One can also verify that for all considered subclasses in $\mathcal{D}$ except the subclass $\mathcal{D}_{10}^{=}$, the coefficients $\tau_{2 j-1}$ vanish, and so the asymptotic expansion (12) contains only the powers of $n^{-1}$. We have prepared the Maple ${ }^{\mathrm{TM}}$ program computing symbolically the coefficients $\tau_{j}$ of (12), in the cases of all six subclasses in $\mathcal{D}$; download the file tvcf-bilequ.mw (or its HTML and PDF version) from the page http://www.rafalnowak.pl/publications/tvcf. Namely, one can get the following asymptotic expansion of the tail $u_{n}$ :

$$
u_{n}= \begin{cases}\tau_{-1} n^{1 / 2}+\tau_{0}+\tau_{1} n^{-1 / 2}+\ldots & \text { if } \mathrm{CF} \in \mathcal{D}_{10}^{=}, \\ \tau_{-2} n+\tau_{0}+\tau_{2} n^{-1}+\ldots & \text { if } \mathrm{CF} \in \mathcal{D}_{10}^{\neq} \cup \mathcal{D}_{11} \cup \mathcal{D}_{20}^{=} \cup \widetilde{\mathcal{D}}_{21}, \\ \tau_{-4} n^{2}+\tau_{-2} n+\tau_{0}+\tau_{2} n^{-1}+\ldots & \text { if } \mathrm{CF} \in \mathcal{D}_{20}^{\neq} .\end{cases}
$$

In order to find the solution $\left\{u_{n}\right\}$, we consider both roots of the quadratic equation (13). Each root determines the solution $X_{n}$ of (10) having the asymptotic representation as in (14). In Lemma 3.1 we give the asymptotic representation of the derived product $I_{n}$.

Table 2 Coefficients $\alpha, \beta, \gamma$

\begin{tabular}{llll}
\hline Subclass & $\alpha$ & $\beta$ & $\gamma$ \\
\hline $\mathcal{D}_{10}^{\bar{\prime}}$ & $q_{0}^{\prime}$ & 0 & $-p_{-1} q_{0}$ \\
$\mathcal{D}_{10}^{\neq}$ & $q_{0}^{\prime}$ & $p_{-1}-p_{-1}^{\prime}$ & 0 \\
$\mathcal{D}_{11}^{\prime}$ & $q_{-1}^{\prime}$ & $q_{-1} q_{-1}^{\prime}$ & 0 \\
$\mathcal{D}_{20}^{\bar{E}}$ & $q_{0}^{\prime}$ & $p_{-2}+p_{-1}-p_{-1}^{\prime}$ & $-p_{-2} q_{0}$ \\
$\mathcal{D}_{20}^{\neq}$ & $q_{0}^{\prime}$ & $p_{-2}-p_{-2}^{\prime}$ & 0 \\
$\widetilde{\mathcal{D}}_{21}^{\prime}$ & $q_{-1}^{\prime}$ & $p_{-2}-p_{-2}^{\prime}+q_{-1} q_{-1}^{\prime}$ & $-p_{-2}^{\prime} q_{-1}$ \\
\hline
\end{tabular}


Lemma 3.1 Let $\left\{X_{n}\right\}$ be a solution of (10), having the asymptotic representation as in (14), and let $Y_{n}=a_{n+1} /\left(b_{n+1}+X_{n+1}\right)$. The product $I_{n}=X_{n} Y_{n}$ has the following asymptotic expansion:

1. In the case of $\mathcal{D}_{10}^{=}$:

$$
I_{n}=p_{-1} n-\frac{p_{-1} q_{0}}{\tau_{-1}} \sqrt{n}+\mathcal{O}(1)
$$

2. In the case of $\mathcal{D}_{10}^{\neq}$:

$$
I_{n}= \begin{cases}p_{-1}^{\prime} n+\mathcal{O}(1) & \text { if } \tau_{-2}=0, \\ p_{-1} n+\mathcal{O}(1) & \text { otherwise; }\end{cases}
$$

3. In the case of $\mathcal{D}_{11}$ :

$$
I_{n}= \begin{cases}\frac{p_{-1} p_{-1}^{\prime}}{q_{-1} q_{-1}^{\prime}}+\mathcal{O}\left(n^{-1 / 2}\right) & \text { if } \tau_{-2}=0, \\ q_{-1} q_{-1}^{\prime} n^{2}+\mathcal{O}(n) & \text { otherwise; }\end{cases}
$$

4. In the case of $\mathcal{D}_{20}^{=}$:

$$
I_{n}=p_{-2} n^{2}+\left(p_{-1}-p_{-2}-\frac{p_{-2} q_{0}}{\tau_{-2}}\right) n+\mathcal{O}(1) ;
$$

5. In the case of $\mathcal{D}_{20}^{\neq}$:

$$
I_{n}= \begin{cases}p_{-2}^{\prime} n^{2}+\mathcal{O}(n) & \text { if } \tau_{-4}=0 \\ p_{-2} n^{2}+\mathcal{O}(n) & \text { otherwise }\end{cases}
$$

6. In the case of $\widetilde{\mathcal{D}}_{21}$ :

$$
I_{n}=\frac{p_{-2} \tau_{-2}}{q_{-1}+\tau_{-2}} n^{2}+\mathcal{O}\left(n^{3 / 2}\right) .
$$

Proof Roughly speaking, the proof follows from the comparison of the asymptotic representation of the possible solutions of the recurrence (10). All the parts of the thesis need to be considered separately. To complete the proof we use the asymptotic expansions (7) and (14). In the cases of $\mathcal{D}_{10}^{\neq}, \mathcal{D}_{11}, \mathcal{D}_{20}^{\neq}$, we study the quadratic equation (13) and consider its both solutions, in order to find the asymptotic expansion of $I_{n}$. The last formula (15f) is well-defined. Indeed, if $\tau_{-2}=-q_{-1}$, then (13) implies $p_{-2} q_{-1}=0$.

Using Lemma 3.1 and the fact that the product $I_{n}$ should be asymptotically smaller in modulus, we can choose a suitable solution $\tau_{-v}$ of (13). In Theorem 3.2, we give this coefficient explicitly. Since the roots of (13) may be zero, we do not call $\tau_{-v}$ the leading coefficient of the expansion (12). Once we have the initial coefficient $\tau_{-\nu}$, we can easily find the next. In Corollary 3.3 we show the beginning of the asymptotic expansion (14) for all subclasses in 
$\mathcal{D}$. In each case, we show only few coefficients, since it is enough to initiate our method for convergence acceleration.

Theorem 3.2 The initial coefficient $\tau_{-v}$ of the asymptotic expansion (12) of the tail $u_{n}$ is the following root of the quadratic equation (13):

1. In the case of $\mathcal{D}_{10}^{=}$:

$$
\tau_{-1}:=\operatorname{sgn}\left(\Re \frac{q_{0}^{\prime} \sqrt{p_{-1} q_{0} / q_{0}^{\prime}}}{p_{-1}}\right) \sqrt{p_{-1} q_{0} / q_{0}^{\prime}} ;
$$

2. In the case of $\mathcal{D}_{10}^{\neq}$:

$$
\tau_{-2}:= \begin{cases}0 & \text { if }\left|p_{-1}^{\prime}\right|<\left|p_{-1}\right| \\ \frac{p_{-1}^{\prime}-p_{-1}}{q_{0}^{\prime}} & \text { if }\left|p_{-1}^{\prime}\right|>\left|p_{-1}\right|\end{cases}
$$

3. In the case of $\mathcal{D}_{11}$ :

$$
\tau_{-2}:=0
$$

4. In the case of $\mathcal{D}_{20}^{=}$:

$$
\tau_{-2}:=\frac{-\beta+\operatorname{sgn}\left(\Re\left(\sqrt{\beta^{2}-4 \alpha \gamma} / p_{-2}\right)\right) \sqrt{\beta^{2}-4 \alpha \gamma}}{2 \alpha},
$$

where $\alpha, \beta$ and $\gamma$ are as in Table 2;

5. In the case of $\mathcal{D}_{20}^{\neq}$:

$$
\tau_{-4}:= \begin{cases}0 & \text { if }\left|p_{-2}^{\prime}\right|<\left|p_{-2}\right|, \\ \frac{p_{-2}^{\prime}-p_{-2}}{q_{0}^{\prime}} & \text { if }\left|p_{-2}^{\prime}\right|>\left|p_{-2}\right| ;\end{cases}
$$

6. In the case of $\widetilde{\mathcal{D}}_{21}, \tau_{-2}:=x_{0}$ if the roots $x_{0}, x_{1}$ of the (13) satisfy

$$
\left|\frac{p_{-2}^{\prime}}{q_{-1}^{\prime}}-x_{0}\right|<\left|\frac{p_{-2}^{\prime}}{q_{-1}^{\prime}}-x_{1}\right| \text {. }
$$

Proof One can easily observe that all the formulas (16a)-(16e) express the solutions of the quadratic equation (13). Moreover, the assumptions given in Table 1 , in the cases of $\mathcal{D}_{10}^{=}$and $\mathcal{D}_{20}^{=}$, imply that the real parts in (16a) and (16d) are nonzero (in the case of $\mathcal{D}_{20}^{=}$, observe that $\alpha, \beta, \gamma$, given in Tables 1 and 2 are the same).

To complete the proof, let us observe that we choose the solution $\tau_{-v}$ of (13), such that the product $I_{n}$, mentioned in Lemma 3.1, is asymptotically smaller in modulus.

In the case of $\mathcal{D}_{11}$, taking into account the expansion (15c), we choose the solution $\tau_{-2}=0$. This proves the part 3 of the thesis. The formulas (15b) and (15e) easily imply the parts 2 and 5 . 
In the case of part 1 , we have the following two solutions of (13):

$$
\tau_{-1}^{ \pm}= \pm \sqrt{p_{-1} q_{0} / q_{0}^{\prime}}
$$

Hence, we obtain that the expansion (15a) can be written in the form

$$
I_{n}^{ \pm}=p_{-1} n+q_{0}^{\prime} \tau_{-1}^{\mp} \sqrt{n}+\mathcal{O}(1),
$$

where $I_{n}^{+}, I_{n}^{-}$correspond to the choice $\tau_{-1}^{+}, \tau_{-1}^{-}$in (15a), respectively. Now, we make use of two obvious facts. First, if $s_{n}=a n^{x}+b n^{y}+\mathcal{O}\left(n^{z}\right)$ for any $x>y>$ $z$, then

$$
\left|s_{n}\right|^{2}=|a|^{2} n^{2 x}+2(\Re a \Re b+\Im a \Im b) n^{x+y}+\mathcal{O}\left(n^{\max \{2 y, x+z\}}\right) .
$$

Second, $\operatorname{sgn} \Re(a / b)=\operatorname{sgn}(\Re a \Re b+\Im a \Im b)$ holds for any $a, b \in \mathbb{C}(b \neq 0)$. The former gives us the following asymptotic expansion of the difference $\left|I_{n}^{-}\right|^{2}-\left|I_{n}^{+}\right|^{2}$ :

$$
\left|I_{n}^{-}\right|^{2}-\left|I_{n}^{+}\right|^{2}=v n^{3 / 2}+\mathcal{O}(n)
$$

and the latter yields that $\operatorname{sgn} v=\operatorname{sgn} \Re\left(q_{0}^{\prime}\left(\tau_{-1}^{+}-\tau_{-1}^{-}\right) / p_{-1}\right)$. Of course, if $v<$ 0 then $I_{n}^{-}$has asymptotically smaller modulus, and so we choose $\tau_{-1}=\tau_{-1}^{-}$. Otherwise, we put $\tau_{-1}=\tau_{-1}^{+}$. To complete the proof of the part 1 , it is enough to observe that

$$
\operatorname{sgn} v=\operatorname{sgn}\left(\Re \frac{q_{0}^{\prime} \sqrt{p_{-1} q_{0} / q_{0}^{\prime}}}{p_{-1}}\right) .
$$

Using the similar technique, one can prove the part 4 of the thesis.

In order to complete the proof of the part 6 , let us observe that inequality (16f) is equivalent to

$$
\left|x_{0} q_{-1}^{\prime}-p_{-2}^{\prime}\right|<\left|x_{1} q_{-1}^{\prime}-p_{-2}^{\prime}\right| \text {. }
$$

By multiplying its both sides by $\left|p_{-2} q_{-1} / q_{-1}^{\prime}\right|$, we obtain

$$
\left|p_{-2} q_{-1} x_{0}-\frac{p_{-2} q_{-1} p_{-2}^{\prime}}{q_{-1}^{\prime}}\right|<\left|p_{-2} q_{-1} x_{1}-\frac{p_{-2} q_{-1} p_{-2}^{\prime}}{q_{-1}^{\prime}}\right| .
$$

Now, let us note that $x_{0} x_{1}=-q_{-1} p_{-2}^{\prime} / q_{-1}^{\prime}$ (equal to the quotient $\gamma / \alpha$ in the case $\widetilde{\mathcal{D}}_{21}$; see Table 2 ). Hence, we have

$$
\left|p_{-2} q_{-1} x_{0}+p_{-2} x_{0} x_{1}\right|<\left|p_{-2} q_{-1} x_{1}+p_{-2} x_{0} x_{1}\right|,
$$

and thus

$$
\left|\frac{p_{-2} x_{0}}{q_{-1}+x_{0}}\right|<\left|\frac{p_{-2} x_{1}}{q_{-1}+x_{1}}\right| .
$$

Therefore, the choice $\tau_{-2}:=x_{0}$ implies that $I_{n}$ has asymptotically smaller absolute value (cf. (15f)). 
Corollary 3.3 The tail $u_{n}$ of CF (2) has the following asymptotic expansion (which contains the powers of $n^{-1 / 2}$ only in the case of $\mathcal{D}_{10}^{=}$):

1. In the case of $\mathcal{D}_{10}^{=}$:

$$
u_{n}=\tau_{-1} n^{1 / 2}+\frac{p_{-1}-2\left(p_{0}-p_{0}^{\prime}+q_{0} q_{0}^{\prime}\right)}{4 q_{0}^{\prime}}+\mathcal{O}\left(n^{-1 / 2}\right) ;
$$

2. In the case of $\mathcal{D}_{10}^{\neq}$:

$$
u_{n}= \begin{cases}\frac{q_{0} p_{-1}^{\prime}}{p_{-1}-p_{-1}^{\prime}}+\mathcal{O}\left(n^{-1}\right) & \text { if }\left|p_{-1}^{\prime}\right|<\left|p_{-1}\right|, \\ \tau_{-2} n+\frac{p_{-1} q_{0}}{p_{-1}^{\prime}-p_{-1}}+\frac{p_{0}^{\prime}-p_{0}-\tau_{-2} q_{1}^{\prime}}{q_{0}^{\prime}}+\mathcal{O}\left(n^{-1}\right) & \text { otherwise; }\end{cases}
$$

3. In the case of $\mathcal{D}_{11}$ :

$$
u_{n}=\frac{p_{-1}^{\prime}}{q_{-1}^{\prime}}+\mathcal{O}\left(n^{-1}\right)
$$

4. In the case of $\mathcal{D}_{20}^{=}$:

$$
u_{n}=\tau_{-2} n+\mathcal{O}(1)
$$

5. In the case of $\mathcal{D}_{20}^{\neq}$:

$$
u_{n}= \begin{cases}\mathcal{O}(1) & \text { if }\left|p_{-2}^{\prime}\right|<\left|p_{-2}\right|, \\ \tau_{-4} n^{2}+\frac{p_{-1}^{\prime}-p_{-1}-\tau_{-4} q_{1}^{\prime}}{q_{0}^{\prime}} n+\mathcal{O}(1) & \text { otherwise }\end{cases}
$$

6. In the case of $\widetilde{\mathcal{D}}_{21}$ :

$$
\begin{aligned}
u_{n}= & \tau_{-2} n \\
& +\frac{q_{0} p_{-2}^{\prime}+q_{-1} p_{-1}^{\prime}-\left(q_{0} q_{-1}^{\prime}+q_{-1} q_{0}^{\prime}+p_{-1}-p_{-2}^{\prime}-p_{-1}^{\prime}\right) \tau_{-2}-\left(q_{-1}^{\prime}+q_{0}^{\prime}\right) \tau_{-2}^{2}}{2 \tau_{-2} q_{-1}^{\prime}+p_{-2}-p_{-2}^{\prime}+q_{-1} q_{-1}^{\prime}} \\
& +\mathcal{O}\left(n^{-1}\right) .
\end{aligned}
$$

\section{Method}

In this section, we present the method for convergence acceleration of $\mathrm{CFs} \in \mathcal{D}$ (see (8)). It is based on the obvious fact: if CF is convergent to $V$, then all its tails $f^{(m)}$ are also convergent, and

$$
S_{m}\left(f^{(m)}\right)=V .
$$

Since $S_{m}(\omega)$ is the linear fractional transformation in $\omega$ (cf. (3)), the modified approximant $S_{m}\left(\omega_{m}\right)$ may be a very efficient approximation of $V$ if $\omega_{m}$ is a good approximation of the tail $f^{(m)}$. 
The main objective of our method is to derive good approximations of the tails $u_{n}$ without computing the coefficients $\tau_{j}$ of (14) explicitly. We compute the table of approximations $u_{n}^{(j)}$ satisfying

$$
\left(u_{n}^{(j+1)}-u_{n}\right) /\left(u_{n}^{(j)}-u_{n}\right) \rightarrow 0 \quad \text { when } \quad n \rightarrow \infty .
$$

Similar concept was used in 'improvement machine'-method of Lorentzen and Waadeland [5], [7, Section 6] (see also [9, p. 227] for more details), and later by Paszkowski [11]. However, 'improvement machine' cannot be applied to all the subclasses in $\mathcal{D}$ and, as numerical examples show, is less efficient than ours.

The following technique is very similar to the one presented in [10], but we deal with more subclasses of CFs and consider only their odd tails $u_{n}$, whose asymptotic expansion can contain powers of $n^{-1 / 2}$.

\subsection{Step of iteration}

Now, we describe a single step, say $j$-th, of the method in detail. Let us assume that we know approximations $u_{n}^{(j)}$ of the tails $u_{n}$, such that

$$
\begin{aligned}
u_{n}^{(j)}= & \tau_{-\nu} n^{\nu / 2}+\tau_{1-\nu} n^{\nu / 2-1 / 2}+\ldots+\tau_{r_{j}-1} n^{1 / 2-r_{j} / 2} \\
& +\varsigma_{0} n^{-r_{j} / 2}+\varsigma_{1} n^{-r_{j} / 2-1 / 2}+\ldots
\end{aligned}
$$

(cf. (12)), where $r_{j} \geq 0$ is an integer number and $\varsigma_{0}, \varsigma_{1}, \ldots$ are some complex numbers. This means that

$$
\delta_{n}^{(j)}:=u_{n}^{(j)}-u_{n}=\mathcal{O}\left(n^{-r_{j} / 2}\right),
$$

We say that approximations $u_{n}^{(j)}$ are of the order $r_{j} / 2$. Now, we define new approximations

$$
v_{n}^{(j)}:=\frac{a_{n}^{\prime}}{b_{n}^{\prime}+\frac{a_{n+1}}{b_{n+1}+u_{n+1}^{(j)}}}
$$

(cf. (9)) and note that they are expressed using the known values. One can verify that modified approximants $S_{2 n-1}\left(v_{n}^{(j)}\right), S_{2 n+1}\left(u_{n+1}^{(j)}\right)$ are equal. Nevertheless, we can use both $u_{n}^{(j)}$ and $v_{n}^{(j)}$ to obtain new approximations $u_{n}^{(j+1)}$ of higher order. To do this, we use the same idea as in [10]. The main result is given in Theorem 4.1. Let us remark that for all subclasses in $\mathcal{D}$ except the subclass $\mathcal{D}_{10}^{=}$, the values $r_{j}$ are even (cf. (14)), and so the orders of the approximations $u_{n}^{(j)}$ are integer for consecutive values of $j$.

Theorem 4.1 Let the approximations $u_{n}^{(j)}$ of the tails $u_{n}$ satisfy (19) with $r_{j}$ such that

$$
r_{j} \geq \begin{cases}0 & \text { if } C F \in \mathcal{D}_{20}^{=} \cup \mathcal{D}_{20}^{\neq}, \\ 1 & \text { if } C F \in \mathcal{D}_{10}^{=}, \\ 2 & \text { otherwise. }\end{cases}
$$


Let $v_{n}^{(j)}$ be as in (20), and let auxiliary functions $\psi_{n}^{(j)}, \varphi_{n}^{(j)}$ be given by

$$
\psi_{n}^{(j)}:=\frac{a_{n+1} a_{n}^{\prime}}{\left(a_{n+1}+b_{n+1} b_{n}^{\prime}+b_{n}^{\prime} u_{n+1}^{(j)}\right)^{2}}, \quad \varphi_{n}^{(j)}:=1+\frac{r_{j}}{2 n} .
$$

Then the approximations $u_{n}^{(j+1)}$ defined by

$$
u_{n}^{(j+1)}:=\frac{\varphi_{n}^{(j)} v_{n}^{(j)}-\psi_{n}^{(j)} u_{n}^{(j)}}{\varphi_{n}^{(j)}-\psi_{n}^{(j)}}
$$

satisfy

$$
u_{n}^{(j+1)}-u_{n}=\mathcal{O}\left(n^{-r_{j+1} / 2}\right),
$$

where $r_{j+1}=r_{j}+2 \theta$ and

$$
\theta:= \begin{cases}1 & \text { if } C F \in \mathcal{D}_{10}^{=} \cup \mathcal{D}_{20}^{=}, \\ 2 & \text { if } C F \in \mathcal{D}_{10}^{\neq} \cup \mathcal{D}_{20}^{\neq} \cup \widetilde{\mathcal{D}}_{21}, \\ 4 & \text { if } C F \in \mathcal{D}_{11}\end{cases}
$$

Proof Roughly speaking, the idea of the proof is to find a relationship between $\delta_{n}^{(j)}$ and $\varepsilon_{n}^{(j)}$, where

$$
\varepsilon_{n}^{(j)}:=v_{n}^{(j)}-u_{n}
$$

First, from (9) and (20) we obtain

$$
\varepsilon_{n}^{(j)}=\psi_{n} \delta_{n+1}^{(j)},
$$

where $\psi_{n}$ is given by

$$
\psi_{n}:=\frac{a_{n+1} a_{n}^{\prime}}{\left(a_{n+1}+b_{n+1} b_{n}^{\prime}+b_{n}^{\prime} u_{n+1}\right)\left(a_{n+1}+b_{n+1} b_{n}^{\prime}+b_{n}^{\prime} u_{n+1}^{(j)}\right)} .
$$

Second, we use the formula relating $\delta_{n+1}^{(j)}$ and $\delta_{n}^{(j)}$ :

$$
\delta_{n+1}^{(j)}=\left(1-\frac{r}{2 n}\right) \delta_{n}^{(j)}+\mathcal{O}\left(n^{-r / 2-\xi}\right), \quad \text { where } \xi:= \begin{cases}\frac{3}{2} & \text { if } \mathrm{CF} \in \mathcal{D}_{10}^{=}, \\ 2 & \text { otherwise }\end{cases}
$$

here and later we omit the lower index $j$ in $r$. This can be verified by substituting $n+1$ in place of $n$ in (19) and using simple algebra. Observe that number $\xi$ is common for all subclasses in $\mathcal{D}$ except the subclass $\mathcal{D}_{10}^{=}$; it follows from the asymptotic expansion (14).

Third, let us observe some properties of function $\psi_{n}^{(j)}$ given in (22). The assumption (21) implies that all the asymptotic expansions in Corollary 3.3 hold also for $u_{n}^{(j)}$, i.e. after replacing $u_{n}$ by $u_{n}^{(j)}$. Using this and the asymptotic 
representations of $a_{n}, a_{n}^{\prime}, b_{n}, b_{n}^{\prime}$ (see (7)), one can find the expansion of numerator and denominator of $\psi_{n}^{(j)}$, and finally obtain that

$$
\psi_{n}^{(j)}=\Theta\left(n^{-\rho}\right), \quad \rho:= \begin{cases}2, & \text { in the case of } \mathcal{D}_{11}, \\ 0, & \text { in all other cases }\end{cases}
$$

where $\Theta\left(n^{\mu}\right)$ has the following meaning:

$$
\Theta\left(n^{\mu}\right)=c n^{\mu}+\mathcal{O}\left(n^{\mu-1 / 2}\right) \quad \text { for some constant } c \neq 0 .
$$

The (29) needs some comment only in the cases of $\mathcal{D}_{10}^{\neq}, \mathcal{D}_{20}^{\neq}$and $\widetilde{\mathcal{D}}_{21}$. Clearly, for all this subclasses, the leading coefficient in the denominator of $\psi_{n}^{(j)}$ does not vanish due to choice of $\tau_{-v}$ (see Theorem 3.2). Now, we use the definition (27) and get

$$
\psi_{n}-\psi_{n}^{(j)}=\frac{a_{n+1} a_{n}^{\prime} b_{n}^{\prime}}{\left(a_{n+1}+b_{n+1} b_{n}^{\prime}+b_{n}^{\prime} u_{n+1}\right)\left(a_{n+1}+b_{n+1} b_{n}^{\prime}+b_{n}^{\prime} u_{n+1}^{(j)}\right)^{2}} \delta_{n+1}^{(j)} .
$$

Using the same arguments as before and the fact that $\delta_{n+1}^{(j)}=\mathcal{O}\left(n^{-r / 2}\right)(\mathrm{cf} .(19))$, we have

$$
\psi_{n}-\psi_{n}^{(j)}=\mathcal{O}\left(n^{-r / 2-\varrho}\right), \quad \varrho:= \begin{cases}1, & \text { in the cases of } \mathcal{D}_{10}^{=}, \mathcal{D}_{10}^{\neq} \text {and } \widetilde{\mathcal{D}}_{21}, \\ 3, & \text { in the case of } \mathcal{D}_{11}, \\ 2, & \text { in the cases of } \mathcal{D}_{20}^{=} \text {and } \mathcal{D}_{20}^{\neq} .\end{cases}
$$

Hence, the (26) can be written in the form

$$
\varepsilon_{n}^{(j)}=\left(\psi_{n}^{(j)}+\mathcal{O}\left(n^{-r / 2-\varrho}\right)\right) \delta_{n+1}^{(j)}=\psi_{n}^{(j)} \delta_{n+1}^{(j)}+\mathcal{O}\left(n^{-r-\varrho}\right) .
$$

Multiplying its both sides by $\varphi_{n}^{(j)}$, we get, after some simple algebra, the relation

$$
\varphi_{n}^{(j)} \varepsilon_{n}^{(j)}-\psi_{n}^{(j)} \delta_{n}^{(j)}=\mathcal{O}\left(n^{-r / 2-\xi-\rho}\right)+\mathcal{O}\left(n^{-r-\varrho}\right) .
$$

Since $\frac{r}{2}+\xi+\rho \leq r+\varrho($ cf. (21)), this can be written as follows:

$$
\varphi_{n}^{(j)} \varepsilon_{n}^{(j)}-\psi_{n}^{(j)} \delta_{n}^{(j)}=\mathcal{O}\left(n^{-r / 2-\zeta}\right),
$$

where $\zeta:=\xi+\rho>0$. Further, let us observe that approximations $u_{n}^{(j+1)}$, given in (23), can be found as a solution of the following equation, derived from (30) by replacing right-hand side by zero and $u_{n}$ (followed by $\delta_{n}^{(j)}$ and $\varepsilon_{n}^{(j)}$ ) by $u_{n}^{(j+1)}$ :

$$
\varphi_{n}^{(j)}\left(v_{n}^{(j)}-u_{n}^{(j+1)}\right)-\psi_{n}^{(j)}\left(u_{n}^{(j)}-u_{n}^{(j+1)}\right)=0 .
$$

Subtracting this formula from (30) yields

$$
\left(\varphi_{n}^{(j)}-\psi_{n}^{(j)}\right)\left(u_{n}^{(j+1)}-u_{n}\right)=\mathcal{O}\left(n^{-r / 2-\zeta}\right) .
$$


To complete the proof of the thesis, it is enough to show that

$$
\varphi_{n}^{(j)}-\psi_{n}^{(j)}=\Theta\left(n^{-\eta}\right), \quad \text { where } \quad \eta:= \begin{cases}\frac{1}{2}, & \text { in the case of } \mathcal{D}_{10}^{=}, \\ 1, & \text { in the case of } \mathcal{D}_{20}^{=}, \\ 0, & \text { in all other cases. }\end{cases}
$$

Then, by dividing the (31) by (32), we obtain the formulas (24) and (25).

In order to prove (32), let us determine the leading coefficients, say $\sigma_{i}$, of the asymptotic expansion of $\varphi_{n}^{\prime}-\psi_{n}^{\prime}$ :

$$
\varphi_{n}^{(j)}-\psi_{n}^{(j)}=\sigma_{0}+\sigma_{1} n^{-1 / 2}+\sigma_{2} n^{-1}+\ldots
$$

From (14) it immediately follows that the coefficients $\sigma_{2 i+1}(i \geq 0)$ may be nonzero only in the case of $\mathcal{D}_{10}^{=}$. The leading coefficients of the asymptotic expansion in (33) can be easily found by use of (7) and (12), and the definitions of $\varphi_{n}^{(j)}, \psi_{n}^{(j)}$. Namely, after some simple algebra, we have

- For $\mathrm{CF} \in \mathcal{D}_{10}^{=}: \quad \sigma_{0}=0, \quad \sigma_{1}=\frac{2 \tau_{-1} q_{0}^{\prime}}{p_{-1}}$;

- For $\mathrm{CF} \in \mathcal{D}_{10}^{\neq}: \quad \sigma_{0}=1-\frac{p_{-1} p_{-1}^{\prime}}{\left(p_{-1}+\tau_{-2} q_{0}^{\prime}\right)^{2}}$;

- For $\mathrm{CF} \in \mathcal{D}_{11}: \quad \sigma_{0}=1$;

- For $\mathrm{CF} \in \mathcal{D}_{20}^{=}: \quad \sigma_{0}=\sigma_{1}=0, \sigma_{2}=\frac{2 \tau_{-2} q_{0}^{\prime}+p_{-1}-p_{-1}^{\prime}}{p_{-2}}+\frac{r}{2}+2$;

- For $\mathrm{CF} \in \mathcal{D}_{20}^{\neq}: \quad \sigma_{0}=1-\frac{p_{-2} p_{-2}^{\prime}}{\left(p_{-2}+\tau_{-4} q_{0}^{\prime}\right)^{2}}$;

- For CF $\in \widetilde{\mathcal{D}}_{21}: \quad \sigma_{0}=1-\frac{p_{-2} p_{-2}^{\prime}}{\left(p_{-2}+q_{-1} q_{-1}^{\prime}+\tau_{-2} q_{-1}^{\prime}\right)^{2}}$.

Now we show that all the above nontrivial coefficients do not vanish. In order to do this, we use Theorem 3.2. If $\mathrm{CF} \in \mathcal{D}_{10}^{=}$, then $\sigma_{1} \neq 0$, since $\tau_{-1} \neq 0$. In the cases of $\mathcal{D}_{k 0}^{\neq}(k \in\{1,2\}), \sigma_{0}$ is equal to $1-p_{k}^{\prime} / p_{k}$, if $\tau_{-k}=0$, and to $1-p_{k} / p_{k}^{\prime}$, otherwise. The cases of $\mathcal{D}_{20}^{=}$and $\widetilde{\mathcal{D}}_{21}$ need quite longer explanation. Namely, let us suppose that $\sigma_{2}=0$ for some $r \in \mathbb{N} \cup\{-1,0\}$, in the case of $\mathcal{D}_{20}^{=}$. Then we have

$$
\frac{1}{2} \geq-\frac{r}{2}=\frac{2 \tau_{-2} q_{0}^{\prime}+p_{-1}-p_{-1}^{\prime}}{p_{-2}}+2 .
$$

Let us denote

$$
\varkappa:=\frac{\sqrt{\beta^{2}-4 \alpha \gamma}}{p_{-2}},
$$

where $\alpha, \beta, \gamma$ are given in Table 2 (see the subclass $\mathcal{D}_{20}^{=}$). From Theorem 3.2 we obtain

$$
2 \tau_{-2} q_{0}^{\prime}+p_{-1}-p_{-1}^{\prime}=-p_{-2}+\operatorname{sgn}(\Re \varkappa) \sqrt{\beta^{2}-4 \alpha \gamma},
$$

and hence the inequality (34) can be written in the form

$$
\frac{1}{2} \geq-\frac{r}{2}=1+\operatorname{sgn}(\Re \varkappa) \varkappa .
$$


We conclude that $\varkappa$ is a real number satisfying $\frac{1}{2} \geq 1+|\varkappa| \geq 1$, which is a contradiction.

In the case of $\widetilde{\mathcal{D}}_{21}$, let us put

$$
z:=\left(p_{-2}+q_{-1} q_{-1}^{\prime}+q_{-1}^{\prime} \tau_{-2}\right)^{2}-p_{-2} p_{-2}^{\prime} .
$$

One can verify that

$$
(2 z-\Delta)^{2}=\Delta\left(p_{-2}+p_{-2}^{\prime}+q_{-1} q_{-1}^{\prime}\right)^{2},
$$

where $\Delta$ is discriminant of the quadratic equation (13), satisfied by $\tau_{-2}$. In the case of subclass $\widetilde{\mathcal{D}}_{21}$, we have obviously $\Delta \neq 0$ (see Table 1 ). Suppose that $\sigma_{0}=0$. Using the following simple algebra:

$$
\begin{aligned}
4 p_{-2} p_{-2}^{\prime} & =\left(p_{-2}+p_{-2}^{\prime}+q_{-1} q_{-1}^{\prime}\right)^{2}-\Delta \\
& =\left(\Delta\left(p_{-2}+p_{-2}^{\prime}+q_{-1} q_{-1}^{\prime}\right)^{2}-\Delta^{2}\right) / \Delta,
\end{aligned}
$$

yields $z=0$, and thus $(2 z-\Delta)^{2}=\Delta^{2}$. This implies that $p_{-2} p_{-2}^{\prime}=0$, which is a contradiction.

\subsection{Algorithm}

Now, we summarize all the previous results and present the algorithm for convergence acceleration of $\mathrm{CFs} \in \mathcal{D}$. It constructs a two-dimensional array $u_{n}^{(j)}$ of the approximations of the tails $u_{n}=f^{(2 n-1)}$. We use Corollary 3.3, in order to obtain the initial approximations $u_{n}^{(0)}$, and thus we have $u_{n}^{(0)}-u_{n}=$ $\mathcal{O}\left(n^{-r_{0} / 2}\right)$, where

$$
r_{0}:= \begin{cases}0, & \text { in the cases of } \mathcal{D}_{20}^{=} \text {and } \mathcal{D}_{20}^{\neq}, \\ 1, & \text { in the case of } \mathcal{D}_{10}^{=}, \\ 2, & \text { in other cases }\end{cases}
$$

(cf. (21)). The main part of the algorithm is the iteration which improves, in each step, the accuracy of approximations $u_{n}^{(j)}$ of the tails $u_{n}$. For consecutive values of $j=0,1,2, \ldots$, we use Theorem 3.2 with approximations $u_{n}^{(j)}$ and obtain the approximations $u_{n}^{(j+1)}$. This process yields the following relation for approximations $u_{n}^{(j)}$ :

$$
u_{n}^{(j)}-u_{n}=\mathcal{O}\left(n^{-r_{j} / 2}\right), \quad j=0,1, \ldots,
$$

where

$$
r_{j}=r_{0}+2 j \theta
$$

and $\theta$ is given in (25). Before we present the algorithm, let us observe some fact simplifying a bit the calculations. Namely, it is enough to use $\psi_{n}^{(0)}$ instead of $\psi_{n}^{(j)}$ in the formula (23). One can verify that all the proof of Theorem 4.1 remains valid, and thus the relation (35) holds true. 
Algorithm 4.2 Consider the CF (2) belonging to $\mathcal{D}$ (see (8))

Initial step. Let the initial approximations $u_{n}^{(0)}$ be given as in Corollary 3.3 with the terms $\mathcal{O}(\cdot)$ removed.

Main loop. Compute the approximations $u_{n}^{(j)}$ using the following formula:

$$
u_{n}^{(j+1)}:=\frac{\varphi_{n}^{(j)} v_{n}^{(j)}-\psi_{n}^{(0)} u_{n}^{(j)}}{\varphi_{n}^{(j)}-\psi_{n}^{(0)}}, \quad n \geq 1, j \geq 0,
$$

where $v_{n}^{(j)}, \psi_{n}^{(0)}$ and $\varphi_{n}^{(j)}$ are given in (20) and (22) (with $r_{j}$ as in (36)), respectively.

Let us remark that the computations of Algorithm 4.2 are organized so that array $u_{n}^{(j)}$ is upper triangular. If we want to compute the approximation $u_{i}^{(j)}$, then we need to start with $j+1$ initial approximations $u_{n}^{(0)}(n=i, i+1, \ldots, i+$ $j$ ). So, the total cost of computing $u_{i}^{(j)}$ is proportional to $j^{2}$.

Let us also note that Algorithm 4.2 is almost fully rational, i.e. only arithmetical operations are used in the main loop. Only in the case of of $\mathcal{D}_{10}^{=}$ the initial calculations need to compute square roots. We also observe that in the cases of $\mathrm{CF} \in \mathcal{D}_{k 0}^{\neq}(k \in\{1,2\})$ with $\left|p_{-k}^{\prime}\right|>\left|p_{-k}\right|$, it is better to apply Algorithm 4.2 to the first tail $f^{(1)}$ of CF which is of the form (2), since then we get the case of $\left|p_{-k}^{\prime}\right|<\left|p_{-k}\right|$, which simplifies the initial calculations.

\section{Numerical results}

In this section, we illustrate some applications of Algorithm 4.2. We give examples of some mathematical functions having continued fraction expansions of the form (2). We apply our method in order to approximate their value.

Consider a two-variant $\mathrm{CF}$ of the form (2), belonging to the class $\mathcal{D}$. In some cases, one may approximate its value using the following classic methods:

(A) Computing classical approximants $S_{n}(0)$ for sufficiently large $n$,

(B) Applying the methods of [7] to even (odd) part of CF,

(C) Applying the methods of [10] to even (odd) part of CF,

(D) Using equivalent transformation in order to obtain limit 2-periodic CF, and using the techniques given in [7],

(E) Using the recent methods of Paszkowski [12];

for more details on numerical computation of CFs see, e.g., [9, Section 5].

Since the convergence of $\mathrm{CF} \in \mathcal{D}$ may be very slow, the first idea should be rather the last resort in order to compute its value.

The methods (B) and (C) require to derive even (odd) part of $\mathrm{CF}$ (see, e.g., [9, Theorem 2.19-2.20, pp. 86-87]). After that, some equivalent transformation (see, e.g., [9, Theorem 2.14, p. 77]) into a limit periodic CF (case (B)) or into an one-variant $\mathrm{CF} \in \mathcal{C}_{20} \cup \mathcal{C}_{21}$ (case (C)) may be needed. However, for some 
subclasses in $\mathcal{D}$, this process leads to the case that the methods (B) and (C) are not applicable. This can be verified using simple analysis considering the even (odd) contraction of $\mathrm{CF} \in \mathcal{D}$. We summarize all the cases in Remark 5.1. We use there the notation for subclasses $\mathcal{I}_{0} \subset \mathcal{C}_{20}$ and $\mathcal{I}_{1} \subset \mathcal{C}_{21}$, introduced in [10, Eqs. 2.2 and 2.3]; see also, e.g., [7, p. 73] for the definition of CFs of loxodromic, elliptic, parabolic and identity type.

Remark 5.1 The even (odd) contraction, applied to $\mathrm{CF} \in \mathcal{D}$, yields as follows:

1. In the cases of $\mathcal{D}_{10}^{=}$and $\mathcal{D}_{20}^{=}$: even (odd) part is equivalent to some $\mathrm{CF} \in$ $\mathcal{C}_{21} \backslash \mathcal{I}_{1}$; further equivalent transformation yields a limit periodic $\mathrm{CF}$ of parabolic type.

2. In the cases of $\mathcal{D}_{10}^{\neq}$and $\mathcal{D}_{20}^{\neq}$: even (odd) part is equivalent to some $\mathrm{CF} \in \mathcal{I}_{1}$, and so the further equivalent transformation gives a limit periodic CF of loxodromic type.

3. In the case of $\mathcal{D}_{11}$ : even (odd) part belongs to $\in \mathcal{C}_{22}$, and so neither to the class $\mathcal{I}_{0}$ nor $\mathcal{I}_{1}$; further equivalent transformation yields a limit periodic $\mathrm{CF}$ of loxodromic type.

4. In the case of $\widetilde{\mathcal{D}}_{21}$ : if $p_{-2}=p_{-2}^{\prime}$, then even (odd) part is equivalent to $\mathrm{CF} \in \mathcal{I}_{1}$ which can be transformed equivalently into a limit periodic $\mathrm{CF}$ of loxodromic type.

In the case of the classic methods (B), the most efficient one is 'improvement machine' of Lorentzen and Waadeland (see [5], [7, Section 6] or [9, p. 227]). However their idea can be applied only to CFs of loxodromic type. On the other hand, the methods of [10] (case (C)) concern subclasses $\mathcal{I}_{0}$ and $\mathcal{I}_{1}$ containing CFs of elliptic and loxodromic type, respectively. So, taking into account the Remark 5.1, the question of the convergence acceleration of $\mathrm{CF} \in \mathcal{D}_{10}^{=} \cup \mathcal{D}_{20}^{=}$is very subtle. According to [7], in the case of limit periodic continued fraction $\mathbf{K}\left(c_{n} / d_{n}\right)$ of parabolic type, one can always use so-called 'fixed point' method (see also [9, p. 219]), i.e. computing modified approximants $S_{n}(-1 / 2)$, provided that, without loss of generality, $c_{n} \rightarrow-1 / 4$ and $d_{n} \rightarrow 1$. But, in order to accelerate its convergence, there are some additional conditions required (see, e.g., [7, Eq. 4.10]). In Examples 5.2 and 5.6 we show a class of CFs (belonging to $\mathcal{D}_{10}^{=}$and $\mathcal{D}_{20}^{=}$) having even (odd) part of parabolic type, for which 'fixed point' method does not work, as well as 'improvement machine' and the methods (C), of course. In this paper, in the case of methods (C), we consider only the 'plus method', described in [10, Algorithm 4.3, p. 310], since it usually gives the best results.

The techniques (D) are quite similar to the case (B), since the application of them depend on the type of limit periodic CF. Again, 'improvement machine' can be applied only in the case of CF of loxodromic type. Other cases need more careful handling.

The methods (E) are based on analytical transformation of the tails of CF. However, they can be applied only in the case of $\mathcal{D}_{10}^{\neq}$(even or odd contraction 
is needed) and $\mathcal{D}_{20}^{=}$, provided that numerators and denominators $a_{n}, a_{n}^{\prime}, b_{n}, b_{n}^{\prime}$ are polynomials in $n$.

Summarizing, all the methods (B)-(E) of computing CFs do not cover the considered class $\mathcal{D}$ of CFs. Moreover, neither of them handle the case of $\mathcal{D}_{10}^{=}$, i.e. the case of $\mathrm{CF}$ with tails having the asymptotic expansion in powers of $n^{-1 / 2}$.

In the sequel, we give a series of examples involving $\mathrm{CFs} \in \mathcal{D}$ and showing the application of Algorithm 4.2. We give additional remarks on computing their value by use of the classic methods (A)-(E), and, if it is possible, we show a comparison.

Before we begin, let us fix some notation to measure the accuracy of the method. Consider the $\mathrm{CF} \in \mathcal{D}$, convergent to the value $V$. Let acc $(x)$ denote the number of exact significant decimal digits of $x$ in $V$ :

$$
\operatorname{acc}(x):=-\log _{10}\left|1-\frac{x}{V}\right| .
$$

We use the following notation:

$$
d_{m}:=\operatorname{acc}\left(S_{m}(0)\right), \quad d_{n}^{(j)}:=\operatorname{acc}\left(S_{2 n-1}\left(u_{n}^{(j)}\right)\right),
$$

where $u_{n}^{(j)}$ is obtained by Algorithm 4.2. Let us remark that $u_{n}^{(j)}$ is the approximation of the tail $u_{n}=f^{(2 n-1)}$ of $\mathrm{CF}$, and that is why we analyse the modified approximant $S_{2 n-1}\left(u_{n}^{(j)}\right)$ (cf. (17)). We call the number $d_{n}^{(j)}$ accuracy of $u_{n}^{(j)}$. In all numerical examples, we observed that it is increasing in $j$, unless it reaches the peak level, which can be, in some cases, more or less the half of the arithmetic precision in a given computer algebra system. We used Maple ${ }^{\mathrm{TM}}$ system with 128-digit (decimal) arithmetic precision.

We did investigations involving all the six subclasses in $\mathcal{D}$. In all the cases, Algorithm 4.2 is very efficient. Its performance is quite similar for both cases of $\mathcal{D}_{10}^{\neq}$and $\mathcal{D}_{20}^{\neq}$, and hence, in this paper, we do not consider the first one. In the case of $\mathrm{CF} \in \mathcal{D}_{11}$, the convergence is quite fast and so it usually does not need to be accelerated.

In Example 5.2, we show the operation of Algorithm 4.2 in detail. We consider $\mathrm{CF} \in \mathcal{D}_{20}^{=}$and compare the results with the classic methods (A) and (E), since the methods (B)-(D) are not applicable thereat.

In Examples 5.3 and 5.4, we investigate some CFs of the subclass $\widetilde{\mathcal{D}}_{21}$. Using the even contraction we obtain the limit periodic CFs which is of loxodromic type. Therefore, we can apply the classic techniques (B)-(D). The numerical examples show that they are less efficient than Algorithm 4.2.

In Example 5.5, we consider CF given in (5), belonging to $\mathcal{D}_{20}^{\neq}$. Considering the classic methods, only the techniques (A)-(D) can be applied. We compare them with Algorithm 4.2.

Finally, in Example 5.6, we consider $\mathrm{CF} \in \mathcal{D}_{10}^{=}$. According to the formula (25), it is the case containing the CFs hardest to accelerate by Algorithm 4.2. Neither of the classic methods (B)-(E) can be applied then. However, Algorithm 4.2 is very effective. 
Example 5.2 Consider the following expansion involving $\mathrm{CF} \in \mathcal{D}_{20}^{=}$:

$$
\begin{aligned}
\frac{4}{\psi\left(\frac{x+3+v}{4}\right)+\psi\left(\frac{x+3-v}{4}\right)-\psi\left(\frac{x+1+v}{4}\right)-\psi\left(\frac{x+1-v}{4}\right)} \\
=x+\mathbf{K}_{n=1}^{\infty}\left[\frac{\left.(2 n-1)^{2}-v^{2}\right\rfloor}{x}+\frac{\left.\left.(2 n)^{2}\right\rfloor\right]}{x}\right]
\end{aligned}
$$

$(\Re x>0$, cf. [13, p. 33, Eq. 12]); $\psi(x)$ denotes the digamma function: $\psi(x)=$ $\Gamma^{\prime}(x) / \Gamma(x)$. One can verify that it is equivalent to the fraction considered by Paszkowski [12]. Namely, we have

$$
x+\underset{n=1}{\mathbf{K}}\left[\frac{(2 n-1)^{2}-v^{2} \mid}{x}+\frac{(2 n)^{2}}{x}\right]=x+\frac{4}{x} \mathcal{M}\left(\frac{x^{2}}{4},-1, \frac{1-v^{2}}{4}, 0,0\right),
$$

where $\mathcal{M}$ class is defined in [12, p. 215, Eq. 5.8]. The fraction (37) is usually extremely slowly convergent. However, if we use Paszkowski's analytical formulas for the fraction (38), we receive CF which convergence is very fast. We will show that Algorithm 4.2 yields similar performance. One can verify that even part of CF (37) is equivalent to CF of the form $\mathbf{K}\left(\tilde{a}_{n} / 1\right)$, where

$$
\tilde{a}_{n}+\frac{1}{4}=\frac{1}{16}\left(x^{2}-1\right) n^{-2}+\frac{1}{32}\left(3 x^{2}-3-v^{2}\right) n^{-3}+\mathcal{O}\left(n^{-4}\right),
$$

and so it is of parabolic type. This means that we cannot use 'improvement machine' of Lorentzen and Waadeland (see [7], [9, p. 227]), as well as the classic methods (C); cf. Remark 5.1. Let us notice that $\Re x>0$, and so the consider $\mathrm{CF}$ converges by virtue of some generalisation of Worpitzky's theorem; see [16, pp. 45-50] or [9, Theorem 3.30, p. 136].

Let us put $x:=1$ and $v:=1 / 2$. We obtain the terribly slowly convergent CF:

$$
V:=1.327052799890558739735 \ldots=1+\underset{n=1}{\mathbf{K}}\left[\frac{(2 n-1)^{2}-\frac{1}{4} \mid}{\mid}+\frac{(2 n)^{2}}{\mid 1}\right] .
$$

For example, the classical approximant $S_{100}(0)=1.319558 \ldots$ yields $d_{100}=$ 2.25 . One can check that $d_{1000}=3.24$ and $d_{10000}=4.24$, so it seems that the accuracy of the classical approximants is increasing more or less logarithmically. Nevertheless, one can derive very good approximation of its value by use of modified approximants $S_{m}\left(\omega_{m}\right)$ with $\omega_{m} \approx f^{(m)}$. Algorithm 4.2 provides 
the approximations $u_{n}^{(j)}$ of the tails $f^{(2 n-1)}$ of increasing accuracy in $j$, in the sense that

$$
\begin{aligned}
u_{n}^{(0)} & =\tau_{-2} n, \\
u_{n}^{(1)} & =\tau_{-2} n+\tau_{0}+\frac{225}{512} n^{-1}+\mathcal{O}\left(n^{-2}\right), \\
u_{n}^{(2)} & =\tau_{-2} n+\tau_{0}+\tau_{2} n^{-1}+\frac{4125}{16384} n^{-2}+\mathcal{O}\left(n^{-3}\right), \\
u_{n}^{(3)} & =\tau_{-2} n+\tau_{0}+\tau_{2} n^{-1}+\tau_{4} n^{-2}+\frac{3124125}{16777216} n^{-3}+\mathcal{O}\left(n^{-4}\right), \\
& \vdots \\
u_{n}^{(j)} & =u_{n}+\mathcal{O}\left(n^{-j}\right), \quad j \geq 4,
\end{aligned}
$$

where $\tau_{i}$ are the coefficients of the asymptotic expansion of the tail $u_{n}=f^{(2 n-1)}$ (see (14)):

$$
\tau_{-2}=2, \quad \tau_{0}=-\frac{15}{16}, \quad \tau_{2}=\frac{225}{1024}, \quad \tau_{4}=\frac{7425}{131072} .
$$

Let us observe that in each step of iteration, we derive only one (nontrivial) coefficient of this expansion; the coefficients $\tau_{2 i-1}$ vanish for all $i \in \mathbb{N}_{0}$ (cf. (14)). Taking into account the definition (25), the considered case $\mathcal{D}_{20}^{=}$ is one of the hardest to accelerate. However, the approximation $u_{1}^{(10)}$ yields almost 10 decimal digits of the value $V$ :

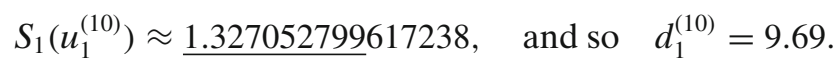

Table 3 shows the accuracies of all the approximations $u_{n}^{(j)}$ needed to be computed in order to obtain the value $u_{1}^{(10)}$. One can observe that accuracy of $u_{1}^{(j)}$ is increasing linearly in $j$. Moreover, it is worth to analyse Fig. 1 depicting the values of $d_{n}^{(j)}$ (accuracies of $u_{n}^{(j)}$ ) for $j=0,1,2,3$ and large values of $n$. It shows that each step of iteration in Algorithm 4.2 is of great importance.

Table 3 Results of Algorithm 4.2 applied to CF (40)

\begin{tabular}{llllllllllll}
\hline$n \backslash j$ & 0 & 1 & 2 & 3 & 4 & 5 & 6 & 7 & 8 & 9 & 10 \\
\hline 1 & 1.24 & 2.40 & 3.21 & 3.96 & 4.70 & 5.44 & 6.21 & 7.02 & 7.86 & 8.75 & 9.69 \\
2 & 1.79 & 3.03 & 3.85 & 4.61 & 5.38 & 6.15 & 6.96 & 7.80 & 8.69 & 9.63 & \\
3 & 2.13 & 3.46 & 4.33 & 5.13 & 5.93 & 6.75 & 7.60 & 8.49 & 9.42 & & \\
4 & 2.38 & 3.78 & 4.70 & 5.55 & 6.40 & 7.26 & 8.15 & 9.08 & & \\
5 & 2.57 & 4.04 & 5.01 & 5.91 & 6.79 & 7.70 & 8.63 & & & \\
6 & 2.73 & 4.25 & 5.27 & 6.21 & 7.14 & 8.08 & & & & \\
7 & 2.86 & 4.44 & 5.50 & 6.48 & 7.44 & & & & & \\
8 & 2.98 & 4.60 & 5.70 & 6.71 & & & & & & \\
9 & 3.08 & 4.74 & 5.88 & & & & & & & \\
10 & 3.17 & 4.87 & & & & & & & & & \\
11 & 3.25 & & & & & & & & & &
\end{tabular}


Fig. 1 Values $d_{n}^{(j)}$ for CF (40)

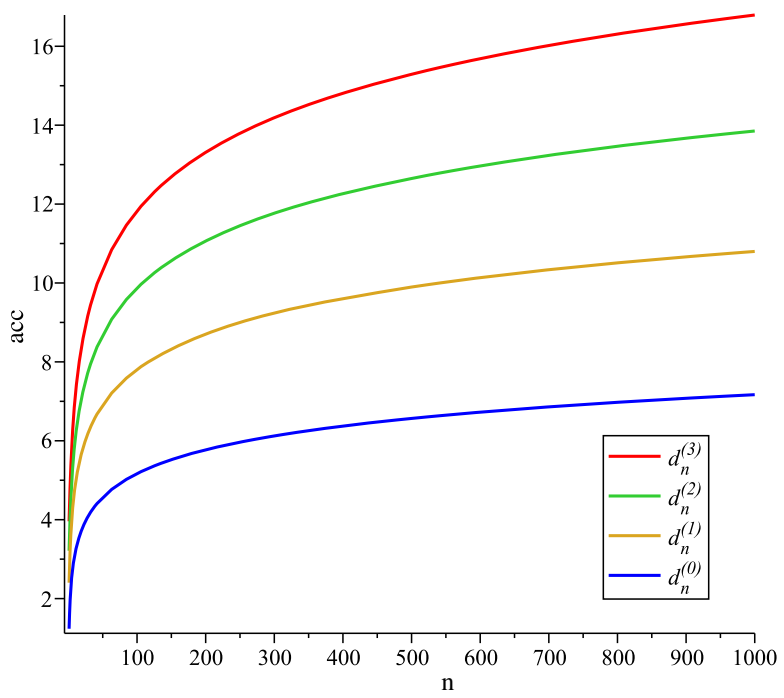

Namely, using single step of iteration is much more productive than using approximations $u_{n}^{(j)}$ for large $n$, provided that $j$ is fixed.

If we use the classic methods (E), i.e. apply Paszkowski's analytical formulas to the fraction (38), we receive CF which is very fast convergent. In the considered case, its classical approximants $\tilde{f}_{n}(n=1,2, \ldots, 10)$ yield the following values of $\operatorname{acc}\left(\tilde{f}_{n}\right)$ :

\section{$2.1,2.9,4.2,5.0,6.3,7.1,8.4,9.2,10.5,11.3$.}

On the other hand, from (39) we have that $a_{n}+\frac{1}{4}=\mathcal{O}\left(n^{-3}\right)$. By virtue of theorem of Thron and Waadeland [15], we obtain that modified approximants $S_{n}(-1 / 2)$ are convergent to the value $V$ faster than classical approximants $S_{n}(0)$, and so 'fixed point' method works. However, it is not very efficient. Indeed, we get the following values of $\operatorname{acc}\left(S_{n}(-1 / 2)\right)$ : 4.52, 6.36, 8.35, 10.34, for $n=10,100,1000,10000$, respectively. In order to obtain 14 decimal digits of $V$ we have to compute $S_{670000}(-1 / 2)$. This should be compared with $d_{1}^{(13)}=$ 14.0 obtained by Algorithm 4.2 after 13 iterations only.

Now, let us put $x:=1 / 2, v:=1 / 2$ in (37). We obtain CF with value $V \approx$ 0.883414269615 and even part being equivalent to $\mathrm{CF}$ of the form $\mathbf{K}\left(\tilde{a}_{n} / 1\right)$, where $\tilde{a}_{n}+1 / 4=-3 / 64 n^{-2}+\mathcal{O}\left(n^{-3}\right)$. One can verify that conditions given by Thron and Waadeland in [15] are not satisfied, so we cannot use their results to accelerate the convergence. Indeed, 'fixed point' method fails, i.e. it gives more or less 0.5 accuracy more than classical approximants $S_{n}(0)$ :

$$
\begin{aligned}
& d_{10}=0.78, \quad \operatorname{acc}\left(S_{10}(-1 / 2)\right)=1.18, \\
& d_{100}=1.21, \quad \operatorname{acc}\left(S_{100}(-1 / 2)\right)=1.67 \text {, } \\
& d_{1000}=1.69, \quad \operatorname{acc}\left(S_{1000}(-1 / 2)\right)=2.16 \text {, } \\
& d_{10000}=2.19, \quad \operatorname{acc}\left(S_{10000}(-1 / 2)\right)=2.66 \text {. }
\end{aligned}
$$


Anyway, one can effectively approximate the value $V$ by use of Algorithm 4.2. We initiate it by approximations $u_{n}^{(0)}=2 n-2 / 3$ (see Corollary 3.3) for $n=$ $1,2, \ldots, 11$, and obtain the upper triangular table of approximations $u_{n}^{(j)}$. The accuracies obtained in the first row-the values of $d_{1}^{(j)}(j=0,1, \ldots, 10)$-are as follows:

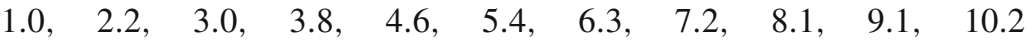

Using Paszkowski's analytical formulas for the fraction (38), we obtain a faster convergent CF (cf. [12, Theorem 5.3, p. 216]). For example, its classical approximants $\tilde{f}_{10}$ and $\tilde{f}_{20}$ yield $\operatorname{acc}\left(\tilde{f}_{10}\right)=11.1$ and $\operatorname{acc}\left(\tilde{f}_{20}\right)=21.6$, while our method (applied to (37)) $-d_{1}^{(10)}=10.2$ and $d_{1}^{(20)}=20.1$, respectively. Let us also remark, that our method is much simpler. Since it constructs the upper triangular table of approximations $u_{n}^{(j)}$ of the tails of $\mathrm{CF}$, the total cost is proportional to the square of the number of initial approximations. However, if we need to compute the value of CF in (37) for many values of parameters $(x, v)$, it is better to use Paszkowski's method, since it constructs, using some analytical transformation, a new $\mathrm{CF}$ which converges very fast.

Example 5.3 Now, let us consider the following $\mathrm{CF} \in \widetilde{\mathcal{D}}_{21}$ :

$$
\frac{x}{\log (1+x)}=1+\underset{n=1}{\mathbf{K}}\left[\frac{n^{2} x \mid}{\mid 2 n}+\frac{n^{2} x}{\mid 2 n+1}\right], \quad x \notin(-\infty,-1] \cup\{0\}
$$

(cf. [13, p. 152, Eq. 7]). We use its even part and obtain $\mathrm{CF} \in \mathcal{C}_{21}$, of the form $\mathbf{K}\left(c_{n} / d_{n}\right)$ with

$$
\begin{aligned}
& c_{n}=-x^{2} n^{2}+2 x^{2} n-\frac{3}{2} x^{2}+\frac{5}{4} x^{2} n^{-1}+\mathcal{O}\left(n^{-2}\right), \\
& d_{n}=(2 x+4) n-2-x+\left(\frac{3 x}{2}+2\right) n^{-1}+\mathcal{O}\left(n^{-2}\right) .
\end{aligned}
$$

One can easily check that it is of loxodromic type and belongs to the subclass $\mathcal{I}_{1}$. Thus, one can use either 'improvement machine' (case (B)) or the classic methods (C). We compare them with Algorithm 4.2.

Let us put complex value of parameter $x=-1.5+0.01$. Notice that it is very close to the ray $(-\infty,-1]$, which is the divergence region of CF (41). It is convergent very slowly then:

$$
d_{10}=-0.3, \quad d_{50}=-0.5, \quad d_{100}=0.1, \quad d_{200}=0.6, \quad d_{300}=0.9 .
$$

We applied 'improvement machine' and 'plus method' to CF $\mathbf{K}\left(c_{n} / d_{n}\right)$ (cf. (42)), as well as Algorithm 4.2 directly to CF (41). All these methods construct upper triangular array of approximations of the tails. We provide all of them only 15 initial approximations. In the case of Algorithm 4.2, we put $u_{n}^{(0)} \approx(-0.9296+0.7106 \mathrm{i}) n+0.3750-0.0250 \mathrm{i}$ in the initial step. Table 4 presents the obtained accuracies. One can observe that improvement machine' yields only 4 decimal digits (cf. the first row), while 'plus method' and Algorithm 4.2-10 and 16 exact significant decimal digits of $V$, respectively. 
Table 4 Computation of (41) with $x=-1.5+0.01$ i by the methods: 'improvement machine' [9, p. 227], method of [10], and Algorithm 4.2

\begin{tabular}{|c|c|c|c|c|c|c|c|c|c|c|c|c|c|c|c|}
\hline$n \backslash j$ & 0 & 1 & 2 & 3 & 4 & 5 & 6 & 7 & 8 & 9 & 10 & 11 & 12 & 13 & 14 \\
\hline \multicolumn{16}{|c|}{ (a) 'Improvement machine': values of $\operatorname{acc}\left(S_{n}\left(w_{n}^{(j)}\right)\right)$} \\
\hline 1 & 0.6 & 0.8 & 1.1 & 1.4 & 1.6 & 1.9 & 2.1 & 2.4 & 2.6 & 2.8 & 3.1 & 3.3 & 3.6 & 3.8 & 4.0 \\
\hline 2 & 1.4 & 1.7 & 2.1 & 2.4 & 2.7 & 3.0 & 3.3 & 3.6 & 3.9 & 4.2 & 4.5 & 4.7 & 5.0 & 5.3 & \\
\hline 3 & 1.8 & 2.3 & 2.7 & 3.1 & 3.5 & 3.8 & 4.2 & 4.5 & 4.8 & 5.1 & 5.4 & 5.8 & 6.1 & & \\
\hline 4 & 2.0 & 2.6 & 3.1 & 3.6 & 4.0 & 4.4 & 4.8 & 5.2 & 5.6 & 5.9 & 6.2 & 6.6 & & & \\
\hline 5 & 2.2 & 2.9 & 3.5 & 4.0 & 4.5 & 4.9 & 5.4 & 5.8 & 6.2 & 6.6 & 6.9 & & & & \\
\hline 6 & 2.4 & 3.1 & 3.8 & 4.3 & 4.9 & 5.4 & 5.8 & 6.3 & 6.7 & 7.1 & & & & & \\
\hline 7 & 2.5 & 3.3 & 4.0 & 4.6 & 5.2 & 5.7 & 6.2 & 6.7 & 7.2 & & & & & & \\
\hline 8 & 2.7 & 3.5 & 4.2 & 4.9 & 5.5 & 6.0 & 6.6 & 7.1 & & & & & & & \\
\hline 9 & 2.8 & 3.6 & 4.4 & 5.1 & 5.7 & 6.3 & 6.9 & & & & & & & & \\
\hline 10 & 2.9 & 3.8 & 4.6 & 5.3 & 6.0 & 6.6 & & & & & & & & & \\
\hline 11 & 3.0 & 3.9 & 4.7 & 5.5 & 6.2 & & & & & & & & & & \\
\hline 12 & 3.0 & 4.0 & 4.9 & 5.7 & & & & & & & & & & & \\
\hline 13 & 3.1 & 4.1 & 5.0 & & & & & & & & & & & & \\
\hline 14 & 3.2 & 4.2 & & & & & & & & & & & & & \\
\hline 15 & 3.2 & & & & & & & & & & & & & & \\
\hline
\end{tabular}

(b) 'Plus method': values of acc $\left(S_{n}\left(t_{n j}\right)\right)$

\begin{tabular}{rrrrrrrrrrrrrrrr}
1 & 0.6 & 1.8 & 2.5 & 3.3 & 4.0 & 4.7 & 5.4 & 6.1 & 6.8 & 7.5 & 8.1 & 8.8 & 9.4 & 10.1 & 10.7 \\
2 & 1.4 & 2.4 & 3.1 & 3.9 & 4.6 & 5.3 & 6.0 & 6.7 & 7.4 & 8.1 & 8.7 & 9.4 & 10.0 & 10.7 & \\
3 & 1.8 & 2.8 & 3.6 & 4.4 & 5.1 & 5.9 & 6.6 & 7.3 & 7.9 & 8.6 & 9.3 & 9.9 & 10.6 & & \\
4 & 2.0 & 3.1 & 4.0 & 4.8 & 5.6 & 6.3 & 7.0 & 7.7 & 8.4 & 9.1 & 9.8 & 10.5 & & \\
5 & 2.2 & 3.3 & 4.3 & 5.1 & 5.9 & 6.7 & 7.4 & 8.1 & 8.9 & 9.6 & 10.2 & & & \\
6 & 2.4 & 3.5 & 4.5 & 5.4 & 6.2 & 7.0 & 7.8 & 8.5 & 9.2 & 10.0 & & & & \\
7 & 2.5 & 3.7 & 4.8 & 5.7 & 6.5 & 7.3 & 8.1 & 8.9 & 9.6 & & & & & \\
8 & 2.7 & 3.9 & 5.0 & 5.9 & 6.8 & 7.6 & 8.4 & 9.2 & & & & & & \\
9 & 2.8 & 4.1 & 5.1 & 6.1 & 7.0 & 7.9 & 8.7 & & & & & & & \\
10 & 2.9 & 4.2 & 5.3 & 6.3 & 7.2 & 8.1 & & & & & & & & \\
11 & 3.0 & 4.3 & 5.5 & 6.5 & 7.4 & & & & & & & & & & \\
12 & 3.0 & 4.4 & 5.6 & 6.7 & & & & & & & & & & & \\
13 & 3.1 & 4.5 & 5.7 & & & & & & & & & & & & \\
14 & 3.2 & 4.6 & & & & & & & & & & & & & \\
15 & 3.2 & & & & & & & & & & & & & & \\
$(\mathrm{c})$ & Algorithm $4.2:$ values of $d_{n}^{(j)}$ & & & & & & & & & \\
1 & 1.1 & 2.4 & 3.4 & 5.0 & 5.8 & 6.9 & 7.9 & 9.0 & 10.0 & 11.1 & 12.1 & 13.1 & 14.2 & 15.2 & 16.2 \\
2 & 1.8 & 3.1 & 4.4 & 5.8 & 6.7 & 7.9 & 8.9 & 10.0 & 11.0 & 12.1 & 13.1 & 14.1 & 15.2 & 16.2 & \\
3 & 2.2 & 3.6 & 5.2 & 6.4 & 7.6 & 8.7 & 9.8 & 10.9 & 11.9 & 13.0 & 14.0 & 15.1 & 16.1 & & \\
4 & 2.5 & 4.0 & 5.8 & 6.9 & 8.3 & 9.4 & 10.6 & 11.6 & 12.8 & 13.8 & 14.9 & 16.0 & & & \\
5 & 2.7 & 4.3 & 6.3 & 7.4 & 8.9 & 10.0 & 11.3 & 12.4 & 13.5 & 14.6 & 15.7 & & & & \\
6 & 2.9 & 4.6 & 6.7 & 7.9 & 9.4 & 10.6 & 11.9 & 13.1 & 14.2 & 15.4 & & & & & \\
7 & 3.0 & 4.8 & 7.1 & 8.3 & 9.9 & 11.1 & 12.4 & 13.7 & 14.9 & & & & & & \\
8 & 3.2 & 5.0 & 7.4 & 8.7 & 10.3 & 11.6 & 13.0 & 14.2 & & & & & & & \\
9 & 3.3 & 5.2 & 7.7 & 9.0 & 10.7 & 12.1 & 13.4 & & & & & & & & \\
10 & 3.4 & 5.4 & 7.9 & 9.3 & 11.0 & 12.5 & & & & & & & & & \\
11 & 3.5 & 5.6 & 8.1 & 9.6 & 11.4 & & & & & & & & & \\
12 & 3.6 & 5.7 & 8.4 & 9.9 & & & & & & & & & & \\
13 & 3.7 & 5.9 & 8.5 & & & & & & & & & & & \\
14 & 3.7 & 6.0 & & & & & & & & & & & & \\
15 & 3.8 & & & & & & & & & & & & & & \\
\hline
\end{tabular}

We did investigations considering $\mathrm{CF}$ in (41) with $\Re x \in[-2,2]$, and $\Im x \in$ $[0.01,2]$. In all the cases Algorithm 4.2 yields better results than the classic methods (B) and (C); we provide all of them 15 initial approximations. 
The biggest difference of accuracies holds for $x=-0.72+0.01 i$. Namely, 'improvement machine', 'plus method' and Algorithm 4.2 yield the accuracy of 14.0, 23.5 and 33.6 exact significant decimal digits of CF value, respectively.

Example 5.4 Let us consider $\mathrm{CF} \in \widetilde{\mathcal{D}}_{21}$ given in (1), and put $x:=\sqrt{2} / 2$. Then, CF converges to $V=\pi /(2 \sqrt{2})$, and so one can use it to approximate the constant $\pi$. Since the convergence of CF is quite fast, it is enough to use its classical approximants. Namely, we have

$$
d_{10}=8.2, \quad d_{20}=15.8, \quad d_{30}=23.5, \quad d_{40}=31.2, \quad d_{50}=38.8 .
$$

Anyway, we apply Algorithm 4.2 to CF

$$
1+\underset{n=1}{\mathbf{K}}\left[\frac{-\left(4 n^{2}-2 n\right) x^{2} \mid}{4 n-1}+\frac{-\left(4 n^{2}-2 n\right) x^{2}}{4 n+1}\right] .
$$

We put $u_{n}^{(0)}=(\sqrt{2}-2) n+(3-\sqrt{2}) / 4$ in the initial step, and obtain $d_{1}^{(9)}=$ 27.5 , which is about 12 digits more than $S_{20}(0)$ yields.

Moreover, if $x$ is a complex number close to the ray $[1,+\infty)$, then convergence of CF in (1) becomes much slower, and the application of convergence acceleration methods is needed. From Remark 5.1, it follows that one can use the classic techniques (B), (C) and (D) in order to compute the CF (1). Numerical examples show that performance of 'improvement machine' is very bad if $x \approx 1$. The methods (C), applied to the even part of (1), gives usually better results. Anyway, the best choice is to use Algorithm 4.2 directly to the two-variant CF (1).

We did investigations considering the complex values of parameter $x \approx 1$. We apply 'improvement machine', 'plus method' and Algorithm 4.2, and compare the obtained accuracies; we provide all of the methods 25 approximations in the initial step.

In the case of 'improvement machine', very often we obtain the upper triangular array of approximations for which the elements in the first row give negative accuracy, and thus none of the exact significant decimal digits of the $\mathrm{CF}$ value. However the next rows may contain the tail approximations having quite good accuracy. Hence, we measure the accuracy of this method by the maximal accuracy obtained by all the elements in the table.

In the cases of 'plus method' and Algorithm 4.2, we measure their accuracies using the last element in the first row of the arrays obtained by these algorithms.

Table 5 shows the accuracies of all the mentioned methods, obtained for the values of parameter $x=0.9990,0.9992,0.9994,0.9996,1+0.00001 \mathrm{i}, 1+$ $0.00005 \mathrm{i}, 1+0.00010 \mathrm{i}, 1+0.00015 \mathrm{i}$. One can observe that if $x$ is closer to 1 , then the lower performance yield the considered methods. Let us observe that for $x=1+0.00001 \mathrm{i}$, the classic methods do not give any information about $\mathrm{CF}$ value; the accuracies are negative. 
Table 5 Accuracies of the methods (B), (C), and Algorithm 4.2 applied to CF (1) with $x \approx 1$

\begin{tabular}{lcccc}
\hline Method $\backslash x$ & 0.9990 & 0.9992 & 0.9994 & 0.9996 \\
\hline Improvement machine & 4.6 & 4.0 & 3.6 & 2.6 \\
Plus method & 6.5 & 5.9 & 2.7 & -0.7 \\
Algorithm 4.2 & 15.6 & 15.0 & 14.2 & 13.2 \\
\hline Method $\backslash x$ & $1+0.00015 \mathrm{i}$ & $1+0.00010 \mathrm{i}$ & $1+0.00005 \mathrm{i}$ & $1+0.00001 \mathrm{i}$ \\
\hline Improvement machine & 0.5 & 0.2 & -0.1 & -0.6 \\
Plus method & 2.8 & 2.4 & 1.3 & -0.4 \\
Algorithm 4.2 & 10.7 & 10.2 & 9.6 & 8.7 \\
\hline
\end{tabular}

Example 5.5 Consider the expansion (5) involving $\mathrm{CF} \in \mathcal{D}_{20}^{\neq}$. In such a case, one cannot use the methods (E). From Remark 5.1, the classic methods (A)-(D) are applicable.

Let us put the following values of parameters: $x:=0.8, k:=0.9$. As we mentioned in Section 1, we are not able to compute the integral in (5) using any current computer algebra systems. So, we apply the method (A) in order to approximate the value of $\mathrm{CF}$. We observe the same 44 significant decimal digits in all the classical approximants $S_{n}(0)$ for $n=1000,1001, \ldots, 10000$, and thus we conclude that $V \approx 0.65478648115337781970888575921$. However, first 100 classical approximants give at most 6.6 digits of $V$. Let us notice that the odd approximants are much better than even ones, since $d_{99}=6.6$ and $d_{100}=3.9$. Hence, we apply the classic methods (B) and (C) to the odd part of CF.

If we initiate Algorithm 4.2 using the approximations $u_{n}^{(0)}=0 \quad(n=$ $1,2, \ldots, 50)$, then we obtain, in a split second, the triangular table of $u_{n}^{(j)}$ $(0 \leq j \leq 24,1 \leq n \leq 50-j)$ giving the accuracy of 24.0 decimal digits of $V$. Namely, the accuracies of the last five approximations in the first row are as follows:

$$
d_{1}^{(20)}=22.1, d_{1}^{(21)}=22.6, d_{1}^{(22)}=23.0, d_{1}^{(23)}=23.5, d_{1}^{(24)}=24.0 .
$$

In the case of the classic methods (B) and (C), applying 'improvement machine' and 'plus method' to the odd part of $\mathrm{CF}$, gives about 9.8 and 17.7 significant decimal digits of $V$, respectively (we provide both methods 50 initial approximations). Let us notice that, in the case of 'improvement machine', the result was not found in the first row of the table; we computed the whole triangular table of approximations and we chose the element giving maximal accuracy. In the case of 'plus method', the best accuracy is obtained by the approximation $t_{1,49}$ which is the last element in the first row of the table computed by this algorithm.

We did investigations involving CF in (5) with $x=0.1,0.2, \ldots, 10.0$ and $k=0.05,0.10,0.15, \ldots, 0.95$. In all the cases we compare the results of Algorithm 4.2 (using 50 initial approximations) with the results of the classic methods (A), (B) and (C). In the case (A), we calculate the classical approximant $S_{99}(0)$, since it gives the best results. In the cases (B) and (C), we apply 'improvement machine' and 'plus method' to the odd part of CF in (5) 
providing both of them 50 approximations in the initial step. Figure 2a depicts the accuracies obtained in the case of $x=0.8$. In all the other cases we obtain similar performance of all the methods, except that 'plus method' becomes worse than 'improvement machine' for $x>4$ and $k=0.95$; see Fig. 2b illustrating the case of $x=10.0$. We observed that Algorithm 4.2 yields better results for all the considered values of parameters $x$ and $k$.

Example 5.6 In this example, we consider the following expansion involving $\mathrm{CF} \in \mathcal{D}_{10}^{=}$:

$$
\begin{aligned}
f(z, \alpha) & :=\left(z^{\alpha-1} e^{z} \int_{z}^{\infty} e^{-v} v^{-\alpha} d v\right)^{-1} \\
& =z+\mathbb{K}_{n=1}^{\infty}\left[\frac{n+\alpha-1}{1}+\frac{n}{z}\right], z>0, \alpha \in \mathbb{R}
\end{aligned}
$$

(cf. [13, p. 103, Eq. 14]). Taking into account the formula (25), this fraction belongs to the subclass which is one of the hardest to accelerate by Algorithm 4.2.

The asymptotic expansion of the $n$-th tail of CF in (43) contains powers of $n^{-1 / 2}$ (see Theorem 3.2), and hence we cannot use the classic methods (E) recent methods of Paszkowski [12] —in order to accelerate the convergence of CF.

Using even contraction of CF in (43), we derive the following one-variant $\mathrm{CF} \in \mathcal{C}_{21}$ :

$$
f(z, \alpha)=z+\frac{\alpha z}{\mid z+1}+\underset{n=2}{\mathbf{K}} \frac{-(n-1)(n+\alpha-1) !}{2 n+z+\alpha-1} .
$$

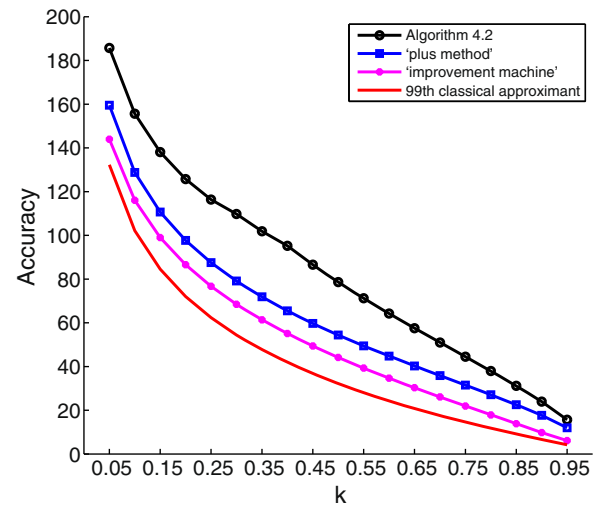

(a) $x=0.8$

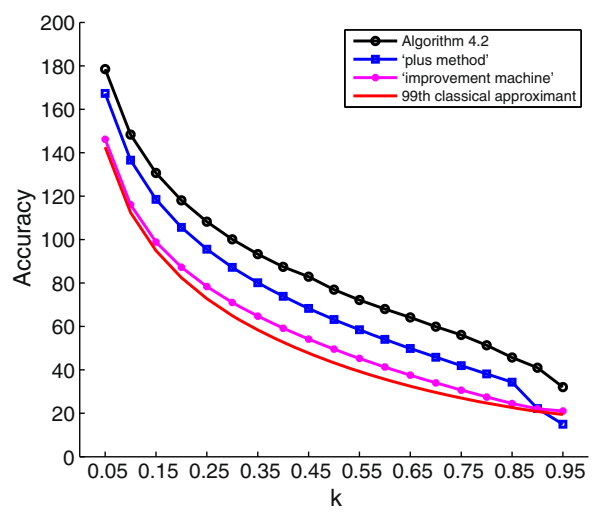

(b) $x=10.0$

Fig. 2 The accuracies of the classic methods and Algorithm 4.2 for CF in (5) 
Unfortunately, we cannot use the methods (C), since the above fraction does not belong to the subclass $\mathcal{I}_{1} \subset \mathcal{C}_{21}$; cf. Remark 5.1. More precisely, the equivalent transformation into limit periodic CF of the form $\mathbf{K}\left(\tilde{a}_{n} / 1\right)$ gives

$$
\tilde{a}_{n}=-\frac{1}{4}+\frac{z}{4} n^{-1}+\mathcal{O}\left(n^{-2}\right)
$$

which is of parabolic type. Therefore, 'fixed point method' is the only applicable technique, in the case of the classic methods (B) and (D).

Let us put $z:=1 / 16$ and $\alpha:=4$. Then CF in (43) is convergent to

$$
V \approx 3.09147726049419952742569567195 \text {. }
$$

We have $d_{10}=0.61, d_{50}=3.02, d_{100}=4.45$ which shows quite slow convergence of the fraction. In fact, (45) does not satisfy the assumptions of Thron and Waadeland [15]. Namely, applying 'fixed point' method to $\operatorname{CF} \mathbf{K}\left(\tilde{a}_{n} / 1\right)$, equivalent to (44), seems to be worthless, since

$$
\begin{array}{ll}
\operatorname{acc}\left(S_{10}(0)\right)=1.45, & \operatorname{acc}\left(S_{10}\left(-\frac{1}{2}\right)\right)=1.45, \\
\operatorname{acc}\left(S_{50}(0)\right)=4.45, & \operatorname{acc}\left(S_{50}\left(-\frac{1}{2}\right)\right)=4.53, \\
\operatorname{acc}\left(S_{100}(0)\right)=6.17, & \operatorname{acc}\left(S_{100}\left(-\frac{1}{2}\right)\right)=6.23 .
\end{array}
$$

This example shows that convergence of considered CF cannot be accelerated using any of the classic methods. In turn, applying Algorithm 4.2 to CF (43) yields, e.g., the approximation $u_{1}^{(79)}$ with accuracy of 26 exact significant decimal digits of $V$. This should be compared to the fact that $S_{n}(0)(n=$ $0,1, \ldots, 160)$ gives at most 6 digits.

Acknowledgement Author wishes to thank Prof. S. Paszkowski for his helpful and constructive comments.

Open Access This article is distributed under the terms of the Creative Commons Attribution License which permits any use, distribution, and reproduction in any medium, provided the original author(s) and the source are credited.

\section{References}

1. Birkhoff, G.D.: Formal theory of irregular linear difference equations. Acta Math. 54, 205246 (1930)

2. Birkhoff, G.D., Trjitzinsky, W.J.: Analytic theory of singular difference equations. Acta Math. 60, 1-89 (1933)

3. Cuyt, A., Petersen, V.B., Verdonk, B., Waadeland, H., Jones, W.B.: Handbook of Continued Fractions for Special Functions. Springer, Dordrecht (2008)

4. Hautot, A.: Convergence acceleration of continued fractions of Poincaré type. Appl. Numer. Math. 4(2-4), 309-322 (1988)

5. Jacobsen, L., Waadeland, H.: Convergence acceleration of limit periodic continued fractions under asymptotic side conditions. Numer. Math. 53(3), 285-298 (1988) 
6. Jones, W.B., Thron, W.J.: Continued Fractions. Analytic Theory and Applications. Encyclopedia of Mathematics and its Applications, vol. 11. Addison-Wesley, London (1980) (distributed by Cambridge University Press, New York)

7. Lorentzen, L.: Computation of limit periodic continued fractions. A survey. Numer. Algorithms 10(1-2), 69-111 (1995)

8. Lorentzen, L., Waadeland, H.: Continued Fractions with Applications. North-Holland, Amsterdam (1992)

9. Lorentzen, L., Waadeland, H.: Continued Fractions. Convergence Theory, vol. 1, 2nd edn. Atlantis Studies in Mathematics for Engineering and Science 1. World Scientific, Hackensack, NJ (2008)

10. Nowak, R.: A method of convergence acceleration of some continued fractions. Numer. Algorithms 41(3), 297-317 (2006)

11. Paszkowski, S.: Convergence acceleration of continued fractions of Poincaré's type 1. Numer. Algorithms 2(2), 155-170 (1992)

12. Paszkowski, S.: Convergence acceleration of some continued fractions. Numer. Algorithms 32(2-4), 193-247 (2003)

13. Perron, O.: Die Lehre von den Kettenbrüchen. Bd II. Analytisch-funktionentheoretische Kettenbrüche. 3. Aufl. B.G. Teubner, Stuttgart (1957)

14. Pincherle, S.: Delle funzioni ipergeometriche e di varie questioni ad esse attinenti. Giorn. Mat. Battaglini 32, 209-291 (1894)

15. Thron, W., Waadeland, H.: Accelerating convergence of limit periodic continued fractions $K\left(a_{n} / 1\right)$. Numer. Math. 34, 155-170 (1980)

16. Wall, H.S.: Analytic Theory of Continued Fractions. Van Nostrand, New York (1948) 